\title{
Mode coupling instability mitigation in friction systems by means of nonlinear energy sinks: Numerical highlighting and local stability analysis
}

\author{
B. Bergeot ${ }^{1, *}$, S. Berger ${ }^{1}$ and S. Bellizzi ${ }^{2}$ \\ ${ }^{1}$ INSA Centre Val de Loire, Université François Rabelais de Tours, LMR EA 2640, Campus de Blois, 3 Rue de la Chocolaterie, \\ CS 23410,41034 Blois Cedex, France \\ ${ }^{2}$ LMA, CNRS, UPR 7051, Centrale Marseille, Aix-Marseille Univ, F-13420 Marseille Cedex 20, France \\ * Corresponding author: baptiste.bergeot@insa-cul.fr
}

\begin{abstract}
In this paper, we study a problem of passive control of friction-induced vibrations due to mode coupling instability in breaking systems. To achieve that, the well-known two degrees of freedom Hultèn's model, which reproduces the typical dynamic behavior of friction systems, is coupled to two ungrounded Nonlinear Energy Sinks (NES). The NES involves an essential cubic restoring force and a linear damping force. First, using numerical simulations it is shown that the suppression or the mitigation of the instability is possible and four steady-state responses are highlighted: complete suppression, mitigation through periodic response, mitigation through strongly modulated response and no suppression of the mode coupling instability. Then the system is analyzed applying complexification-averaging method, the resulting slow-flow is finally analyzed using geometric singular perturbation theory. This analysis allows to explain the observed steady state response regimes and predict some of them. The boundary values of the friction coefficient for some of the transitions between these regimes are predicted. However, the appearance of a three-dimensional super-slow flow subsystem highlights the limitation of the local linear stability analysis of the slow-flow to predict all these boundaries.
\end{abstract}

Keywords: Friction-induced vibration, Passive control, Non linear energy sink, Relaxation oscillations, Strongly modulated response.

\section{Introduction}

Self-excited systems play a key role in numerous industrial applications related to the fields of aeronautics, railways, and cars. Dry friction systems are good examples of these systems (Sinou et al. [2006a,b], Chevennement-Roux et al. [2007], Sinou and Jézéquel [2007], Hervé et al. [2008]). They develop dynamic instabilities related to the friction which are explained in major cases by two main families of mechanisms. The first family explains the instabilities by the variation of the friction coefficient with respect to the relative speed or by a higher static friction coefficient than the dynamic one. The stick-slip is a well-known phenomenon in this context (Van De Velde and De Baets $[1998 b, a])$. The second family attributes the appearance of instabilities to the sprag-slip mechanism and more generally to the mode-coupling phenomenon. In this case, self-excited oscillations may occur even with a constant friction coefficient. In most brake models the instability is due to mode-coupling phenomenon (Fritz et al. [2007], Oden and Martins [1985]) which is studied in this paper. Moreover, it has been shown that the well-known two degrees of freedom Hultèn's model (Hultén $[1997,1993])$ is sufficient to investigate the mode-coupling instability (D'Souza and Dweib [1990], Eriksson and Jacobson [2001], Hoffmann and Gaul [2003], Nechak et al. [2013]). In a nutshell, friction systems and especially the braking systems are subject to dynamic instabilities leading to limit cycle oscillations that may affect their efficiency and the user comfort. Moreover, it is very difficult to design completely stable systems, particularly because of the dispersion of friction laws. It is therefore necessary to attenuate these vibrations. 
The concept Targeted Energy Transfer (TET) is a relatively new passive control method which consists in coupling an essentially nonlinear attachment also named Nonlinear Energy Sink (NES) to an existing primary system prone to unwanted vibrations. TET has been extensively studied numerically, theoretically and more rarely experimentally. The results prove that the NES is very efficient for vibration mitigation (Vakatis et al. [2008]) and noise reduction (Bellet et al. [2010]). Impulsive loading was theoretically analyzed for example by Vakakis and Gendelman [2001] where TET is investigated in terms of resonance capture. Starosvetsky and Gendelman [2008] investigate harmonic forcing where response regimes are characterized in terms of periodic and strongly modulated responses using an asymptotic analysis (multi scale approach) of the averaged flow obtained using the complexification-averaging method (Manevitch [1999]). Ahmadabadi and Khadem [2013] investigate the role of a single degree of freedom NES with nonlinear damping characteristics in annihilating undesired periodic response regimes and simultaneously preserving strongly modulated responses (SMR). The studied system consists in a harmonically excited three degrees of freedom system consisting of two linear coupled oscillators and an NES attached to it. Gourc et al. [2013] use a NES to reduce chatter vibration in turning process. An application of NES as a nonlinear vibration absorber in rotor dynamics can be found in Bab et al. [2014] where the efficiency of a collection of NES is analyzed for vibration mitigation of a rotating system under mass eccentricity force. Bab et al. [2015] investigated the performance of a NES to mitigate vibration of a rotating beam under an external forced. We can also cite Farid and Gendelman [2015] which study the applicability of common pendulum as the NES for mitigation of impulsive excitations. The authors present a theoretical analysis of the damped targeted energy transfer into the pendulum NES from the primary mass with an account of corrections caused by the effect of gravity.

NES are also used to control dynamic instabilities. The possible suppression of the limit cycle oscillations of a Van der Pol oscillator utilizing a NES is demonstrated numerically in Lee et al. [2006]. In Gendelman and Bar [2010] (resp. Domany and Gendelman [2013]), the self-excitation response regimes of a Van der Pol (resp. Van der Pol-Duffing) oscillator with a NES are investigated. An asymptotic analysis of the system related to slow/super-slow decomposition of the averaged flow reveals periodic responses, global bifurcations of different types and basins of attraction of various self-excitation regimes. A series of papers by Lee et al. [2007a,b], Gendelman et al. [2010] demonstrated that a NES coupled to a rigid wing in subsonic flow can partially or even completely suppress aeroelastic instability. In Lee et al. [2007a], the suppression mechanisms are investigated numerically. Several aspects of the suppression mechanisms are validated experimentally in Lee et al. [2007b]. Moreover, an asymptotic analysis is reported in Gendelman et al. [2010] demonstrating the existence of the three passive suppression mechanisms based on TET. Suppression of aeroelastic instability of a general nonlinear multi degree of freedom system has also be considered in Luongo and Zulli [2013]. A theoretical/numerical analysis of the capacity of a NES to control helicopter ground resonance instability (which is a mode-coupling instability) has been performed by Bergeot et al. [2016a,b]. More generally, the discussion on relationship between dimensionality of the super-slow manifold, structure of the fixed points and the observed response regimes is explored in review paper by Gendelman [2011].

In this context, the use of NES appears to be an interesting way to control mode-coupling instability in braking systems. The goal of the paper is therefore to study the effect of coupling two NES to the two degree of freedom model defined by Hultèn. The originality of this paper focuses on two things: (1) to our knowledge, there are no previous studies on this subject for the dry friction system and (2) in the context of the analysis of this kind of systems, the appearance of a three-dimensional super-slow flow subsystem (the vocabulary will be clarified in the paper) highlights the limitation of the local linear stability analysis of the slow-flow to predict all the steady-state response regimes of the system.

The paper is organized as follows. In Sect. 2, the system under study is presented. It consists in a Hultén's model coupled to two ungrounded NES. In Sect. 3, the local stability analysis of the trivial solution for the with-NES and without-NES systems is presented. Using numerical simulations, the Sect. 4 presents some steady-state response regimes which result from the NES attachments. We count four regimes classified into two categories depending on the fact that the trivial solution of the coupled system is stable or not. The simple local stability analysis per- 


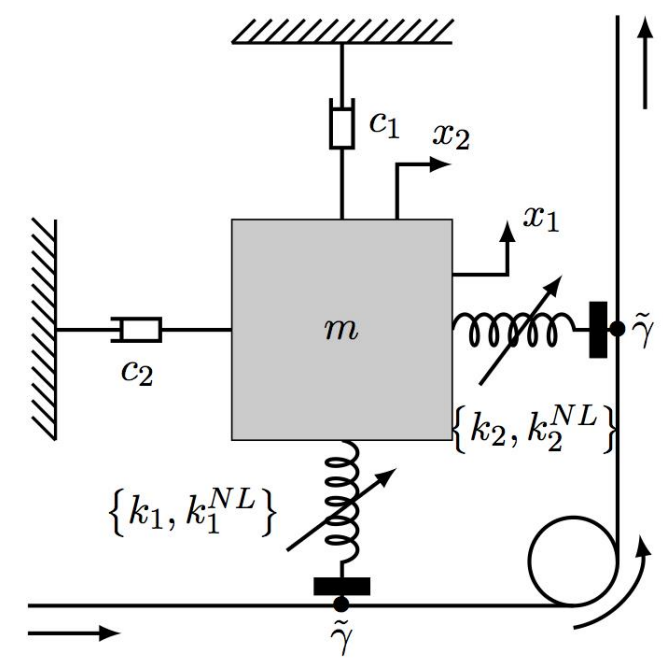

Figure 1: Mechanical system without NES.

formed in Sect. 3 is sufficient to find if the NES are able to to suppress completely the dynamic instability. By contrast, trying to describe and predict the other mechanisms (i.e. when the trivial equilibrium remains unstable despite the presence of the NES), a more technical mathematical development is required. It is performed in Sect. 5 following an analytical procedure based on complexification-averaging method together with geometric singular perturbation theory. Positive results and limitations of this analytical work are discussed in Sect. 6. Finally, additional analysis using numerical simulations and a benchmark of the theoretical results obtained in Sect. 6 are performed in Sect. 7.

\section{System under study}

\subsection{The primary system}

In this paper a simple self-excited system proposed by Hultén [1997, 1993] is used. Hultèn's model reproduces the typical dynamic behavior of friction systems. Therefore, it is sufficient to investigate how passive control of friction-induced vibration due to mode-coupling phenomenon by means of NES can be performed. The simplicity of this model allows to develop analytical expressions in order to better understand the role of the NES attachment.

This model is composed of a mass $m$ held against a moving band; the contact between the mass and the band is modeled by two plates supported by two different springs (see Fig. 1). For the sake of simplicity, it is usually assumed that the mass and band surfaces are always in contact. This assumption may be due to a preload applied to the system. The contact can be expressed by two cubic stiffnesses, see for example Sinou and Jézéquel [2007]. Damping is integrated as shown in Fig. 1. The friction coefficient at contact is assumed to be constant and the band moves at a constant velocity. Then it is assumed that the direction of friction force does not change because the relative velocity between the band speed and $d x_{1} / d t$ or $d x_{2} / d t$ is assumed to be positive. All these assumptions are taken into account in order to study a simple non-linear theoretical 2 degrees-of-freedom system with friction such that the effects of damping on mode coupling instability and the associated analytical developments may be easily investigated. The tangential force $F_{T}$ due to friction contact is assumed to be proportional to the normal force $F_{N}$ as given by Coulomb's law: $F_{T}=\tilde{\gamma} F_{N}$, where $\tilde{\gamma}$ is the friction coefficient. Assuming the normal force $F_{N}$ is linearly related to the displacement of the mass normal to the contact surface, the resulting equations of motion can be expressed as

$$
\begin{gathered}
m \frac{d^{2} x_{1}}{d t^{2}}+c_{1} \frac{d x_{1}}{d t}+k_{1} x_{1}-\tilde{\gamma} k_{2} x_{2}+ \\
k_{1}^{N L} x_{1}^{3}-\tilde{\gamma} k_{2}^{N L} x_{2}^{3}=0 \\
m \frac{d^{2} x_{2}}{d t^{2}}+c_{2} \frac{d x_{2}}{d t}+k_{2} x_{2}+\tilde{\gamma} k_{1} x_{1}+ \\
\tilde{\gamma} k_{1}^{N L} x_{1}^{3}+k_{2}^{N L} x_{2}^{3}=0 .
\end{gathered}
$$

\subsection{Mechanical model with Nonlinear Energy Sinks}

Two identical NES with masses $m_{h}$, linear stiffnesses $k_{h}$, damping coefficients $c_{h}$ and a cubic stiffnesses $k_{h}^{N L}$, are attached on the system in an ungrounded configuration (see Fig. 2). Because a NES is an essentially nonlinear oscillator the linear stiffness $k_{h}$ is assumed to be very smaller than cubic stiffness $k_{h}^{N L}$. This assumption is in agreement with experimental data (see for example Bellet et al. [2010], Gourdon et al. [2007], Kerschen et al. [2007]).

Taking into account the NES displacements $h_{1}(t)$ and $h_{2}(t)$, the equations of motion (1) become

$$
\begin{array}{r}
m \frac{d^{2} x_{1}}{d t^{2}}+c_{1} \frac{d x_{1}}{d t}+k_{1} x_{1}-\tilde{\gamma} k_{2} x_{2}+ \\
k_{1}^{N L} x_{1}^{3}-\tilde{\gamma} k_{2}^{N L} x_{2}^{3}+
\end{array}
$$




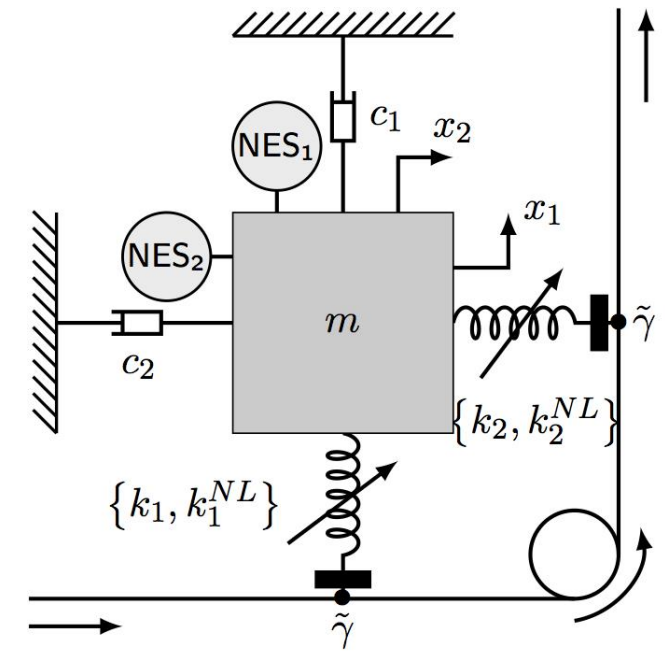

(a)

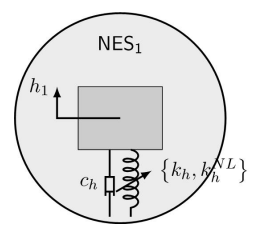

(b)

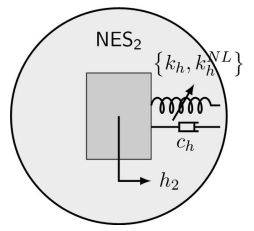

(c)
Figure 2: (a) Mechanical system with NES. (b) Zoom on the $\mathrm{NES}_{1}$. (c) Zoom on the $\mathrm{NES}_{2}$.

$c_{h}\left(\frac{d x_{1}}{d t}-\frac{d h_{1}}{d t}\right)+k_{h}\left(x_{1}-h_{1}\right)+k_{h}^{N L}\left(x_{1}-h_{1}\right)^{3}=0$

$$
\begin{aligned}
m_{h} \frac{d^{2} h_{1}}{d t^{2}}+ & c_{h}\left(\frac{d h_{1}}{d t}-\frac{d x_{1}}{d t}\right)+ \\
& k_{h}\left(h_{1}-x_{1}\right)+k_{h}^{N L}\left(h_{1}-x_{1}\right)^{3}=0
\end{aligned}
$$

$$
\begin{gathered}
m \frac{d^{2} x_{2}}{d t^{2}}+c_{2} \frac{d x_{2}}{d t}+k_{2} x_{2}+\tilde{\gamma} k_{1} x_{1}+ \\
\tilde{\gamma} k_{1}^{N L} x_{1}^{3}+k_{2}^{N L} x_{2}^{3}+ \\
c_{h}\left(\frac{d x_{2}}{d t}-\frac{d h_{2}}{d t}\right)+k_{h}\left(x_{2}-h_{2}\right)+k_{h}^{N L}\left(x_{2}-h_{2}\right)^{3}=0 \\
m_{h} \frac{d^{2} h_{2}}{d t^{2}}+c_{h}\left(\frac{d h_{2}}{d t}-\frac{d x_{2}}{d t}\right)+ \\
k_{h}\left(h_{2}-x_{2}\right)+k_{h}^{N L}\left(h_{2}-x_{2}\right)^{3}=0 .
\end{gathered}
$$

Introducing the following notation $\tilde{\eta}_{i}=c_{i} / \sqrt{m k_{i}}$, $\omega_{i}=\sqrt{k_{i} / m}, \bar{\varphi}_{i}=k_{i}^{N L} / m$ (with $\left.i=1,2\right), \epsilon=$ $m_{h} / m, \bar{\xi}_{h}=k_{h} / m, \tilde{\mu}=c_{h} / \sqrt{m k_{1}}$ and $\bar{\varphi}_{h}=k_{h}^{N L} / m$, Eqs. (2) become

$$
\begin{gathered}
\frac{d^{2} x_{1}}{d t^{2}}+\tilde{\eta}_{1} \omega_{1} \frac{d x_{1}}{d t}+\omega_{1}^{2} x_{1}-\tilde{\gamma} \omega_{2}^{2} x_{2}+ \\
\bar{\varphi}_{1} x_{1}^{3}-\tilde{\gamma} \bar{\varphi}_{2} x_{2}^{3}+ \\
\tilde{\mu} \omega_{1}\left(\frac{d x_{1}}{d t}-\frac{d h_{1}}{d t}\right)+\bar{\xi}_{h}\left(x_{1}-h_{1}\right)+\bar{\varphi}_{h}\left(x_{1}-h_{1}\right)^{3}=0 \\
\epsilon \frac{d^{2} h_{1}}{d t^{2}}+\tilde{\mu} \omega_{1}\left(\frac{d h_{1}}{d t}-\frac{d x_{1}}{d t}\right)+\quad(3 \mathrm{a}) \\
\bar{\xi}_{h}\left(h_{1}-x_{1}\right)+\bar{\varphi}_{h}\left(h_{1}-x_{1}\right)^{3}=0 \\
\tilde{\mu} \omega_{1}\left(\frac{d x_{2}}{d t}-\frac{d h_{2}}{d t}\right)+\bar{\xi}_{h}\left(x_{2}-h_{2}\right)+\bar{\varphi}_{h}\left(x_{2}-h_{2}\right)^{3}=0 \\
\tilde{\gamma} x_{2}^{2}+\tilde{\eta}_{2} \omega_{2} \frac{d x_{2}}{d t}+\omega_{2}^{2} x_{2}+\tilde{\gamma} \omega_{1}^{2} x_{1}+ \\
\epsilon \frac{d^{2} h_{2}}{d t^{2}}+\tilde{\mu} \omega_{1}\left(\frac{d h_{2}}{d t}-\frac{d x_{2}}{d t}\right)+ \\
\bar{\xi}_{h}\left(h_{2}-x_{2}\right)+\bar{\varphi}_{h}\left(h_{2}-x_{2}\right)^{3}=0
\end{gathered}
$$

with $0<\epsilon \ll 1$, assuming that the mass of the NES is small with respect to the mass of the primary system. In theoretical and experimental works devoted to the systems with NES, the mass ratio $\epsilon$ is adopted to stay in a range 0.01-0.1 and this convention will be followed in current work. As it will be demonstrated below, relative smallness of $\epsilon$ is crucial, if one develops the analytic approach to the problem.

In the remaining of this section few notations are introduced in order to obtain a system written into a form which facilitate the theoretical study performed in Sect. 5.

First, changing the time scale from $t$ to $t^{*}=\omega_{1} t$ and noting " . " the derivative with respect to time $t^{*}$, Eqs. (3) take the following form

$$
\begin{aligned}
& \ddot{x}_{1}+\tilde{\eta}_{1} \dot{x}_{1}+x_{1}-\tilde{\gamma}(1-\tilde{a})^{2} x_{2}+ \\
& \tilde{\varphi}_{1} x_{1}^{3}-\tilde{\gamma} \tilde{\varphi}_{2} x_{2}^{3}+\tilde{\mu}\left(\dot{x}_{1}-\dot{h}_{1}\right)+ \\
& \tilde{\alpha}_{1}\left(x_{1}-h_{1}\right)+\tilde{\alpha}\left(x_{1}-h_{1}\right)^{3}=0
\end{aligned}
$$

$$
\begin{gathered}
\epsilon \ddot{h}_{1}+\tilde{\mu}\left(\dot{h}_{1}-\dot{x}_{1}\right)+\tilde{\alpha}_{1}\left(h_{1}-x_{1}\right)+\tilde{\alpha}\left(h_{1}-x_{1}\right)^{3}=0 \\
\left.\ddot{x}_{2}+\tilde{\eta}_{2}(1-\tilde{a}) \dot{x}_{2}+(1-\tilde{a})\right)^{2} x_{2}+\tilde{\gamma} x_{1}+ \\
\tilde{\gamma} \tilde{\varphi}_{1} x_{1}^{3}+\tilde{\varphi}_{2} x_{2}^{3}+\tilde{\mu}\left(\dot{x}_{2}-\dot{h}_{2}\right)+
\end{gathered}
$$




$$
\begin{array}{r}
+\tilde{\alpha}_{1}\left(x_{2}-h_{2}\right)+\tilde{\alpha}\left(x_{2}-h_{2}\right)^{3}=0 \\
(4 \mathrm{c}) \\
\epsilon \ddot{h}_{2}+\tilde{\mu}\left(\dot{h}_{2}-\dot{x}_{2}\right)+\tilde{\alpha}_{1}\left(h_{2}-x_{2}\right)+\tilde{\alpha}\left(h_{2}-x_{2}\right)^{3}=0,
\end{array}
$$

with $\omega_{2} / \omega_{1}=1-\tilde{a}, \tilde{\varphi}_{2}=\bar{\varphi}_{2} / \omega_{1}^{2}, \tilde{\varphi}_{2}=\bar{\varphi}_{2} / \omega_{1}^{2}, \tilde{\alpha}_{1}=$ $\bar{\xi}_{h} / \omega_{1}^{2}$ and $\tilde{\alpha}=\bar{\varphi}_{h} / \omega_{1}^{2}$.

We assume that the parameters $\tilde{\eta}_{1}, \tilde{\eta}_{2}, \tilde{\gamma}, \tilde{\varphi}_{1}, \tilde{\varphi}_{2}, \tilde{\mu}, \tilde{\alpha}$ and $\tilde{a}$ are of order $\epsilon$ (i.e $\tilde{\eta}_{1}, \tilde{\eta}_{2}, \tilde{\gamma}, \tilde{\varphi}_{1}, \tilde{\varphi}_{2}, \tilde{\mu}, \tilde{\alpha}, \tilde{a} \sim O(\epsilon)$, with $\left.0<\epsilon \ll 1\right)$. Moreover, because the linear stiffness of the NES is supposed to be very smaller than the cubic stiffness, it is stated that $\alpha_{1} / \alpha \sim O\left(\epsilon^{2}\right)$.

In order to perform asymptotic analysis in next sections the parameters of the system are rescaled taking into account previous assumptions

$$
\begin{aligned}
\eta_{1} & =\frac{\tilde{\eta_{1}}}{\epsilon} ; & \varphi_{1} & =\frac{\tilde{\varphi}_{1}}{\epsilon} ; & \gamma & =\frac{\tilde{\gamma}}{\epsilon} ; \\
\mu & =\frac{\tilde{\mu}}{\epsilon} ; & \eta_{2} & =\frac{\tilde{\eta_{2}}}{\epsilon} ; & \varphi_{2} & =\frac{\tilde{\varphi}_{2}}{\epsilon} ; \\
a & =\frac{\tilde{a}}{\epsilon} ; & \alpha & =\frac{\tilde{\alpha}}{\epsilon} ; & \alpha_{1} & =\frac{\tilde{\alpha}_{1}}{\epsilon^{3}},
\end{aligned}
$$

with $\eta_{1}, \eta_{2}, \gamma, \varphi_{1}, \varphi_{2}, \mu, a, \alpha, \alpha_{1} \sim O(1)$.

Using rescaled parameters (5), Eqs. (4) become

$$
\begin{aligned}
& \ddot{x}_{1}+\epsilon \eta_{1} \dot{x}_{1}+x_{1}-\epsilon \gamma(1-\epsilon a)^{2} x_{2}+ \\
& \epsilon \varphi_{1} x_{1}^{3}-\epsilon^{2} \gamma \varphi_{2} x_{2}^{3}+\epsilon \mu\left(\dot{x}_{1}-\dot{h}_{1}\right)+ \\
& \epsilon^{3} \alpha_{1}\left(x_{1}-h_{1}\right)+\epsilon \alpha\left(x_{1}-h_{1}\right)^{3}=0
\end{aligned}
$$

$\ddot{h}_{1}+\mu\left(\dot{h}_{1}-\dot{x}_{1}\right)+\epsilon^{2} \alpha_{1}\left(h_{1}-x_{1}\right)+\alpha\left(h_{1}-x_{1}\right)^{3}=0$

$$
\begin{aligned}
& \ddot{x}_{2}+\epsilon \eta_{2}(1-\epsilon a) \dot{x}_{2}+(1-\epsilon a)^{2} x_{2}+\epsilon \gamma x_{1}+ \\
& \epsilon^{2} \gamma \varphi_{1} x_{1}^{3}+\epsilon \varphi_{2} x_{2}^{3}+\epsilon \mu\left(\dot{x}_{2}-\dot{h}_{2}\right)+ \\
& \epsilon^{3} \alpha_{1}\left(x_{2}-h_{2}\right)+\epsilon \alpha\left(x_{2}-h_{2}\right)^{3}=0
\end{aligned}
$$

$\ddot{h}_{2}+\mu\left(\dot{h}_{2}-\dot{x}_{2}\right)+\epsilon^{2} \alpha_{1}\left(h_{2}-x_{2}\right)+\alpha\left(h_{2}-x_{2}\right)^{3}=0$.

System of Eqs. (6) is the Rescaled Hulten's Model including NES (RHM+NES). The remaining of the paper is devoted to the analysis of its steady-state regimes.

\section{Linear stability of the trivial solu- tion}

Using the notation introduced in Sect. 2.2, Eq. (1) reduces to the Rescaled Primary System (RPS)

$$
\begin{gathered}
\ddot{x}_{1}+\epsilon \eta_{1} \dot{x}_{1}+x_{1}-\epsilon \gamma(1-\epsilon a)^{2} x_{2}+ \\
\epsilon \varphi_{1} x_{1}^{3}-\epsilon^{2} \gamma \varphi_{2} x_{2}^{3}=0 \\
\ddot{x}_{2}+\epsilon \eta_{2}(1-\epsilon a) \dot{x}_{2}+(1-\epsilon a)^{2} x_{2}+\epsilon \gamma x_{1}+ \\
\epsilon^{2} \gamma \varphi_{1} x_{1}^{3}+\epsilon \varphi_{2} x_{2}^{3}=0 .
\end{gathered}
$$

We focus the analysis on the capacity of the NES attachments to suppress or mitigate vibrations when the primary system is unstable. That is why in this section the local stability of the trivial equilibrium of the RPS (7) is computed and compared to the local stability of the trivial equilibrium of the RHM+NES (6).

Local stability of the trivial equilibrium is found by looking the sign of the eigenvalues real parts of the Jacobian matrices of the vector functions $\mathbf{F}_{\mathbf{1}}$ and $\mathbf{F}_{2}$ evaluated at the trivial equilibrium, denoted respectively $D \mathbf{F}_{\mathbf{1}}(0)$ and $D \mathbf{F}_{\mathbf{2}}(0)$. The vector functions $\mathbf{F}_{\mathbf{1}}$ and $\mathbf{F}_{\mathbf{2}}$ characterize respectively the RPS (7) and the RHM+NES (6) when they are formally written in state-space form

$$
\dot{\mathbf{X}}=\mathbf{F}_{\mathbf{1}}(\mathbf{X}) \text {, with } \mathbf{X}=\left[\begin{array}{lll}
x_{1} & x_{2} & \dot{x}_{1} \\
\dot{x}_{2}
\end{array}\right]^{t},
$$

and

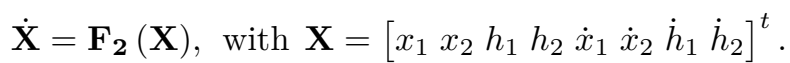

The evolution of the real and imaginary parts of the resulting eigenvalues of the Jacobian $D \mathbf{F}_{\mathbf{1}}(0)$ with respect to the rescaled friction coefficient $\gamma$ is plotted in Fig. 3 and compared to that of the Jacobian matrix $D \mathbf{F}_{\mathbf{2}}(0)$ in Fig. 4 for the following values of the parameters

$$
\begin{aligned}
a & =1, & \eta_{1} & =0.4, & \eta_{2} & =1.2, \\
\epsilon & =0.01, & \varphi_{1} & =5, & \varphi_{2} & =0, \\
\mu & =0.4, & \alpha & =7, & & \alpha_{1}=7 .
\end{aligned}
$$

For the RPS, there are four eigenvalues (two pair of complex conjugate) denoted $\lambda_{i}^{\text {wo }}$ (with $i \in[1,4]$ ), they are represented in Fig. 3, only two of these eigenvalues $\left(\lambda_{1}\right.$ and $\left.\lambda_{3}\right)$ are plotted in Fig. 4 . Due to the 
presence of the NES, four additional eigenvalues are observed compared to the system without NES. Because of the weak coupling between the NES and the primary system, these additional eigenvalues are close to the eigenvalues of the uncoupled linearized equation of motion of the NES. Each linearized equation of motion has two eigenvalues given by the following expression: $\frac{1}{2}\left(-\mu \pm \sqrt{\mu^{2}-4 \alpha_{1} \epsilon^{2}}\right)$. The RHM+NES have therefore eight eigenvalues denoted $\lambda_{i}^{w}$ (with $i \in[1,8])$.

We can notice that the trivial equilibrium is hyperbolic for both the RPS (7) and the RHM+NES (6) regardless the value of $\gamma$, i.e. all the eigenvalues of $D \mathbf{F}_{\mathbf{1}}(0)$ and $D \mathbf{F}_{\mathbf{2}}(0)$ have non zero real parts (except at the Hopf bifurcation points hereafter defined). In this case, The Hartman-Grobman theorem (e.g., Wiggins [1990], Chap. 3) states that in the vicinity of such a hyperbolic equilibrium point, the nonlinear systems (8) and (9) have the same qualitative stability as does the corresponding linear systems. This guarantees the validity of the present local stability analysis ${ }^{1}$.

For both RPS and RHM+NES, as usual the Hopf bifurcation points is defined as the particular value of $\gamma$ for which at least the real part of one of the eigenvalues switches from negative to positive while the real part of the other eigenvalues remains negative. At the Hopf bifurcation points the trivial equilibrium switches from stable to unstable. We notice in Fig. 4 that the presence of the NES shifts the bifurcation to a larger value of $\gamma$. This is the linear effect of the NES due the additional damping $\mu$.

For the RPS the bifurcation point is denoted $\gamma_{b}$ and it is used as the origin for following theoretical study. Therefore, in the remaining of the paper the bifurcation parameter under consideration is the detuning term $\sigma$ defined as follows

$$
\gamma=\gamma_{b}+\sigma
$$

The other parameters are fixed (see Eq. (10)).

For the set of parameters (10), $\gamma_{b}=1.12$ and the bifurcation point of the RHM+NES is equal to $\gamma=$ 1.43 , corresponding to

$$
\sigma=0.31
$$

The parameters (10) are chosen to illustrate with only one set of parameters the potential of the NES to modify the response regimes after the bifurcation

\footnotetext{
${ }^{1}$ In the present paper all fixed points of all system of differential equations are checked to be hyperbolic.
}

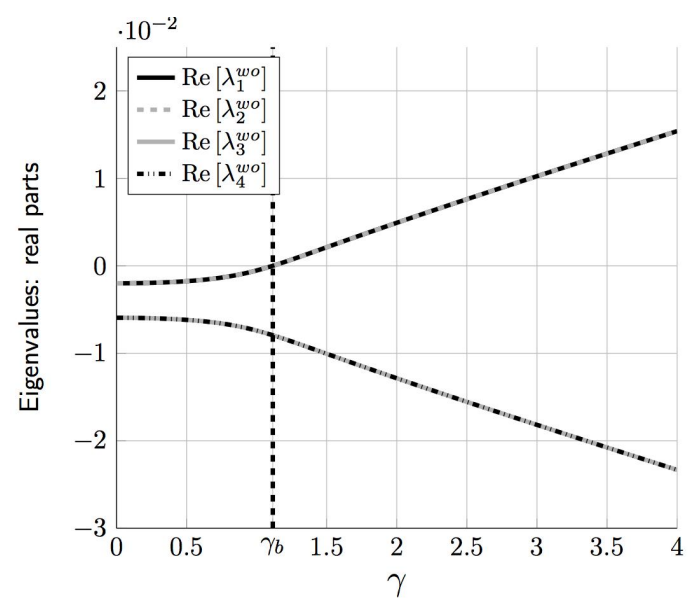

(a) Real parts

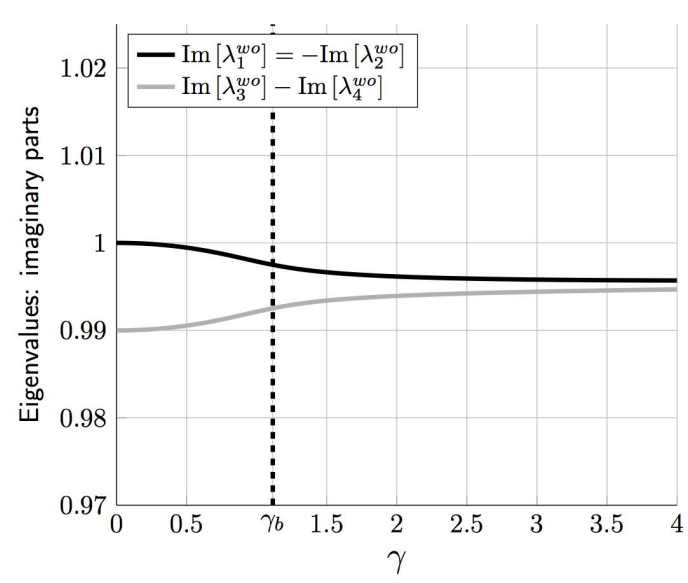

(b) Imaginary parts

Figure 3: Evolution of real and imaginary parts of the eigenvalues of the Jacobian matrix of the vector function $\mathbf{F}_{\mathbf{1}}$ evaluated at the trivial equilibrium. Parameters used: $a=1, \eta_{1}=0.4, \eta_{2}=1.2$ and $\epsilon=0.01 . \gamma_{b}$ is the Hopf bifurcation point.

point. These modified steady-state regimes are presented in the following section.

\section{Possible steady-state response regimes}

The aim of this section is first to present the main steady-state response regimes which may result from the NES attachments and their relevance. For that, the time series $x_{1}\left(t^{*}\right)$ and $x_{2}\left(t^{*}\right)$, resulting from the numerical integration of the RHM+NES, Eqs. (6) and of the RPS, Eqs. (7), are compared in Fig. 5. In both cases, same initial conditions are used, chosen as small perturbation of the trivial solution: $x_{1}(0)=0.05$, $x_{2}(0)=h_{1}(0)=h_{2}(0)=\dot{x}_{1}(0)=\dot{x}_{2}(0)=\dot{h}_{1}(0)=$ 


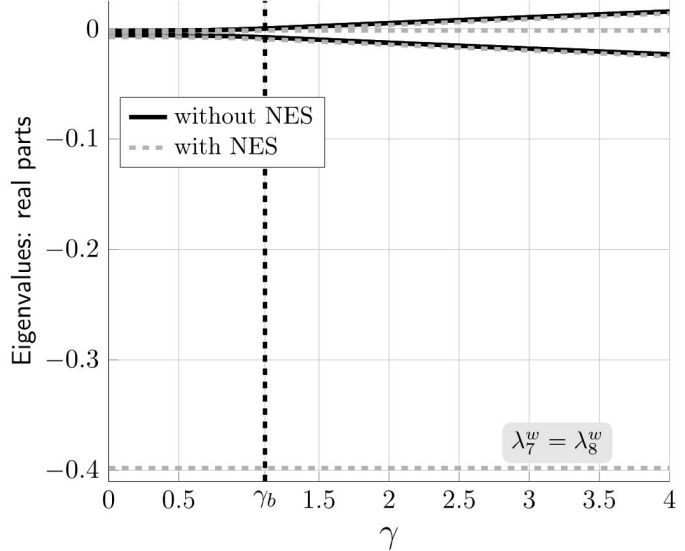

(a) Real parts

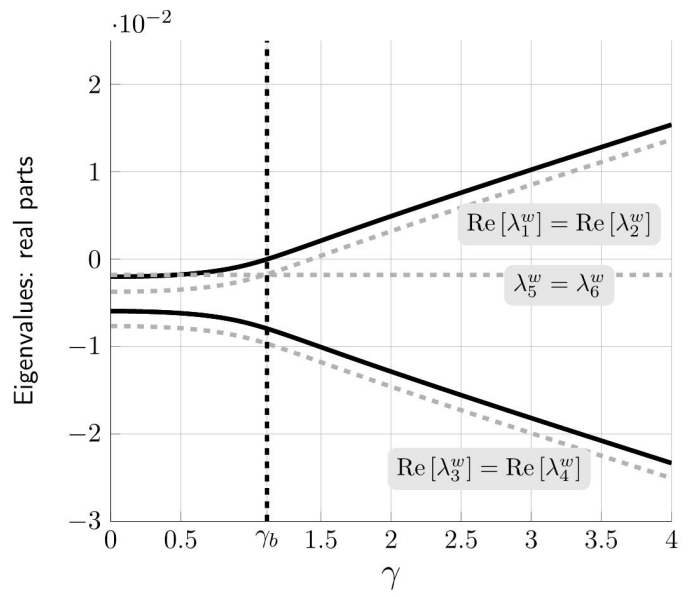

(c) Real parts (zoom)

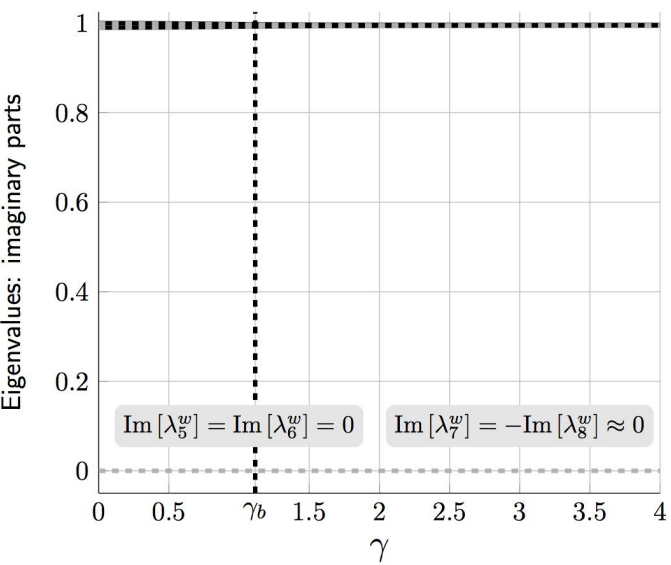

(b) Imaginary parts

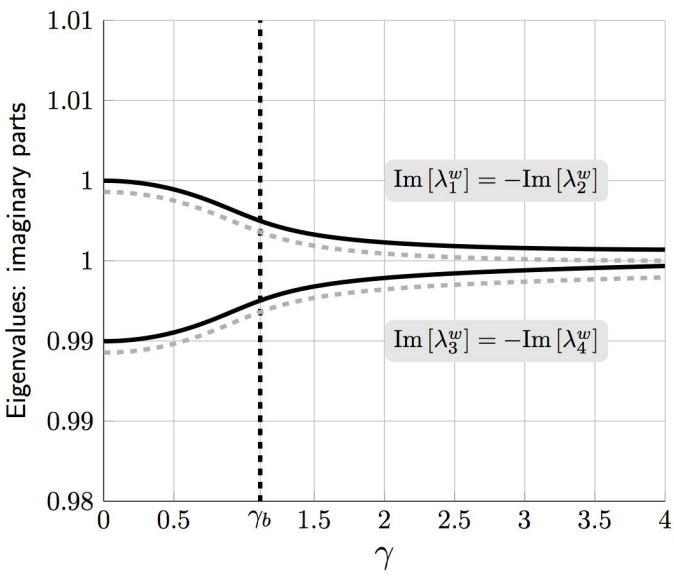

(d) Imaginary parts (zoom)

Figure 4: Evolution of real and imaginary parts of the eigenvalues of the Jacobian matrices of the vector functions $\mathbf{F}_{\mathbf{1}}$ (solid black lines) and $\mathbf{F}_{\mathbf{2}}$ (dashed gray lines) evaluated at the trivial equilibrium. Parameters used: $a=1, \eta_{1}=0.4$, $\eta_{2}=1.2, \epsilon=0.01, \alpha_{1}=7$ and $\mu=0.03$.

$\dot{h}_{2}(0)=0$.

Observing the displacements $x_{1}\left(t^{*}\right)$ and $x_{2}\left(t^{*}\right)$ (solid red line in Fig. 5) of the RHM+NES, four main types of response regimes which may be generated when a NES is attached on the system are highlighted selecting different values of the parameter $\sigma$. They are classified into two categories depending on the fact that the trivial solution of the RHM+NES is stable or not:

\section{- The trivial solution of the RHM+NES is} stable:

- Complete suppression (see. Fig. 5(a)). In this case, the additional damping due to the NES attachment stabilizes the system and the mode-coupling instability is completely suppressed.

- The trivial solution of the RHM+NES is unstable:

- Mitigation through Periodic Response (PR) (see. Fig. 5(b)). In this case, the steadystate response regime is periodic with frequency close to $1^{2}$.

- Mitigation through Strongly Modulated Response (SMR) (see. Fig. 5(c)). In this case, the steady-state response regime is a quasiperiodic regime which exhibits a "fast" component with frequency close to 1 and a

\footnotetext{
${ }^{2}$ This can be shown for example by computing the power spectrum of the steady part of the signal.
} 

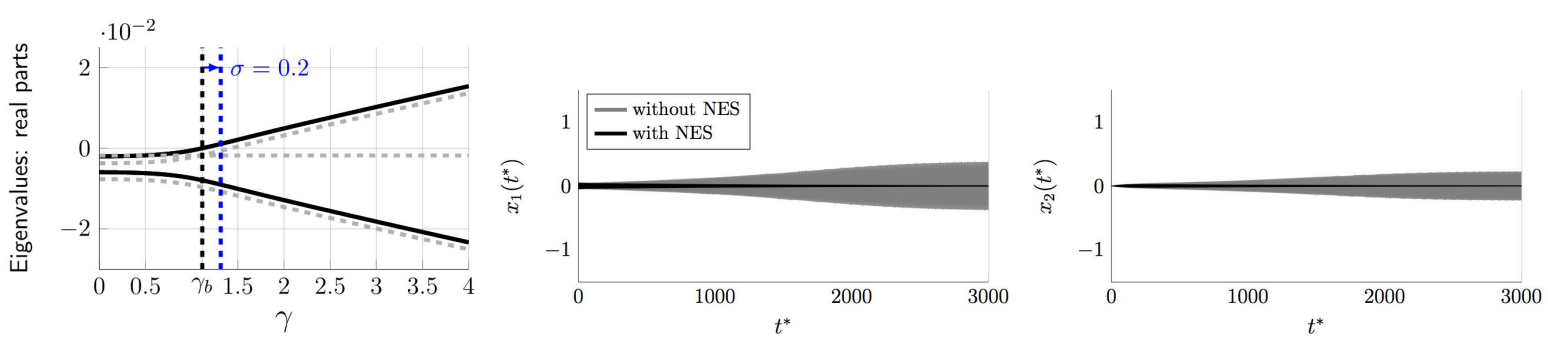

(a) Complete suppression
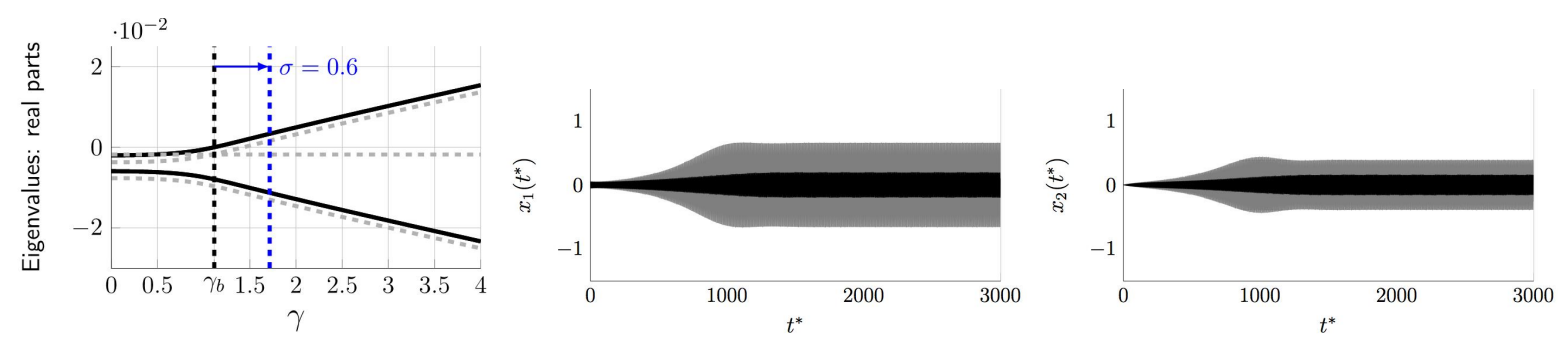

(b) Mitigation: PR
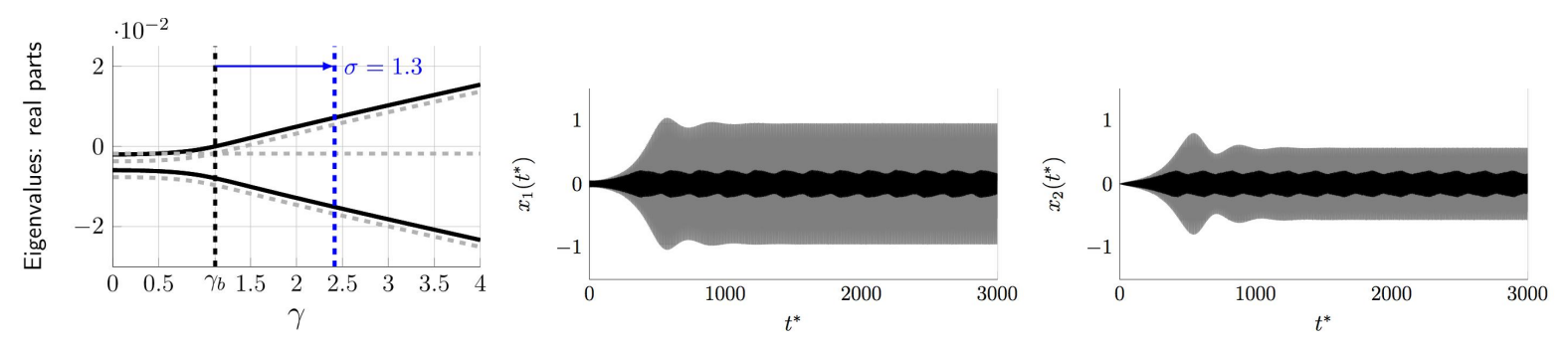

(c) Mitigation: SMR
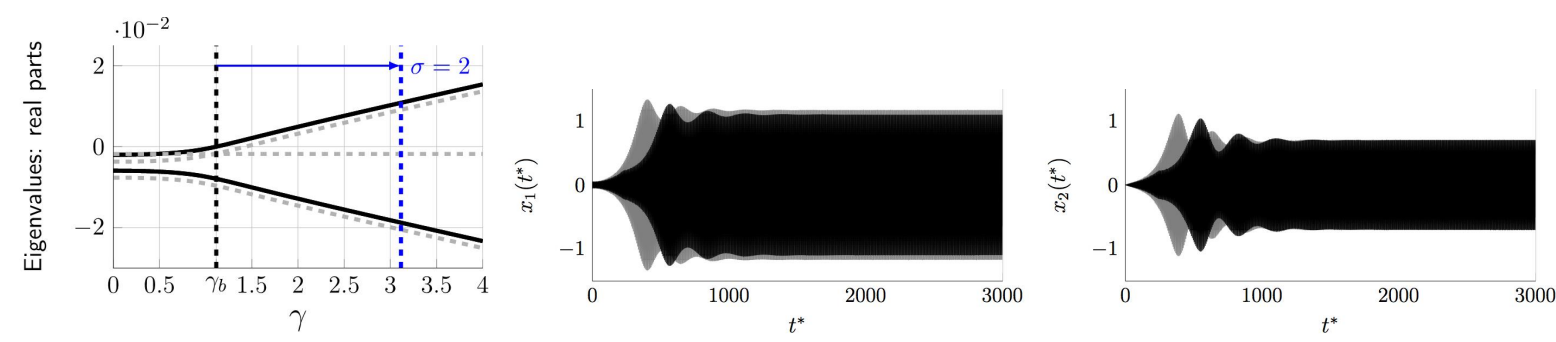

(d) No suppression

Figure 5: Comparison between time series $x_{1}\left(t^{*}\right)$ and $x_{2}\left(t^{*}\right)$ resulting from the numerical integration of the RHM+NES (Eqs. (6)) (solid black line) and $x_{1}\left(t^{*}\right)$ and $x_{2}\left(t^{*}\right)$ resulting of the numerical integration of the RPS (Eqs. (7)) (solid gray line). Set of parameters (10) is used with (a) $\sigma=0.2$, (b) $\sigma=0.6$, (c) $\sigma=1.3$ and (d) $\sigma=2$. On the left, the position of each simulation in the graph representing the real parts of the eigenvalues with respect to $\gamma$ is shown.

"slow" component corresponding to the envelope of the signal. The term "Strongly modulated response" has been introduced by Starosvetsky and Gendelman [2008] for the study of a harmonically forced linear system coupled to a NES.

- No mitigation (see. Fig. 5(d)). The NES is not able to mitigate the instability. Indeed, a periodic regime is observed with an am- plitude close to the amplitude of the system without NES.

These four responses are also observed by Lee et al. [2007a] and study theoretically by Gendelman et al. [2010] in the context of the mitigation of aeroelastic instabilities of a rigid wing in subsonic flow by means of a NES. Furthermore, Bergeot et al. [2016a] observed these responses studying control of helicopter ground resonance instability attaching a NES on the 
fuselage of the helicopter.

Using numerical simulations, the capacity of NES to suppress or mitigate vibrations due dynamic instability in friction systems has been highlighted in this section. Nevertheless, numerical simulations are not sufficient to have a good understanding of the mitigation mechanisms. That is why the RHM+NES is analyzed in the following section.

\section{Asymptotic analysis of the Hulten's Model including NES (RHM+NES)}

The analysis presented in this section is first based on Complexification-Averaging method (CA-X) introduced by Manevitch [1999] and discussed in detail by Vakatis et al. [2008]. The CA-X leads to the determination of the slow-flow of the system. This slow-flow is then analyzed using Geometric Singular Perturbation Theory (GSPT) (Fenichel [1979], Jones [1995], Desroches et al. [2012]).

\subsection{The slow-flow}

First, to simplify the following calculations, it is convenient to introduce barycentric coordinates $u_{i}(t)$ and $v_{i}(t)($ with $i=1,2)$

$$
\begin{array}{ll}
u_{1}=x_{1}+\epsilon h_{1}, & v_{1}=x_{1}-h_{1}, \\
u_{2}=x_{2}+\epsilon h_{2}, & v_{2}=x_{2}-h_{2},
\end{array}
$$

and reciprocally,

$$
\begin{array}{ll}
x_{1}=\frac{u_{1}+\epsilon v_{1}}{\epsilon+1}, & h_{1}=\frac{u_{1}-v_{1}}{\epsilon+1}, \\
x_{2}=\frac{u_{2}+\epsilon v_{2}}{\epsilon+1}, & h_{1}=\frac{u_{2}-v_{2}}{\epsilon+1} .
\end{array}
$$

Using Eqs. (13-16), Eqs. (6) are written as follows

$$
\begin{gathered}
\ddot{u}_{1}+\frac{\eta_{1} \epsilon\left(\dot{u}_{1}+\epsilon \dot{v}_{1}\right)}{\epsilon+1}+\frac{u_{1}+\epsilon v_{1}}{\epsilon+1}- \\
\frac{\epsilon(a \epsilon-1)^{2}\left(\gamma_{b}+\sigma\right)\left(u_{2}+\epsilon v_{2}\right)}{\epsilon+1}+ \\
\frac{\epsilon \varphi_{1}\left(u_{1}+\epsilon v_{1}\right)^{3}}{(\epsilon+1)^{3}}-\frac{\epsilon^{2} \varphi_{2}\left(\gamma_{b}+\sigma\right)\left(u_{2}+\epsilon v_{2}\right)^{3}}{(\epsilon+1)^{3}}=0
\end{gathered}
$$

$$
\begin{gathered}
\frac{\epsilon(a \epsilon-1)^{2}\left(\gamma_{b}+\sigma\right)\left(u_{2}+\epsilon v_{2}\right)}{\epsilon+1}+ \\
\mu(1+\epsilon) \dot{v}_{1}+\alpha(1+\epsilon) v_{1}^{3}+\frac{\epsilon \varphi_{1}\left(u_{1}+\epsilon v_{1}\right)^{3}}{(\epsilon+1)^{3}}- \\
\frac{\epsilon^{2} \varphi_{2}\left(\gamma_{b}+\sigma\right)\left(u_{2}+\epsilon v_{2}\right)^{3}}{(\epsilon+1)^{3}}=0 \\
\ddot{u}_{2}-\frac{\eta_{2} \epsilon(a \epsilon-1)\left(\dot{u}_{2}+\epsilon \dot{v}_{2}\right)}{\epsilon+1}+ \\
\frac{(a \epsilon-1)^{2}\left(u_{2}+\epsilon v_{2}\right)}{\epsilon+1}+\frac{\epsilon\left(\gamma_{b}+\sigma\right)\left(u_{1}+\epsilon v_{1}\right)}{\epsilon+1}+ \\
\frac{\epsilon \varphi_{2}\left(u_{2}+\epsilon v_{2}\right)^{3}}{(\epsilon+1)^{3}}+\frac{\epsilon^{2} \varphi_{1}\left(\gamma_{b}+\sigma\right)\left(u_{1}+\epsilon v_{1}\right)^{3}}{(\epsilon+1)^{3}}=0 \\
\ddot{v}_{2}+\epsilon^{2} \alpha_{1}(1+\epsilon) v_{2}-\frac{\eta_{2} \epsilon(a \epsilon-1)\left(\dot{u}_{2}+\epsilon \ddot{v}_{2}\right)}{\epsilon+1}+ \\
\frac{(a \epsilon-1)^{2}\left(u_{2}+\epsilon v_{2}\right)}{\epsilon+1}+\frac{\epsilon\left(\gamma_{b}+\sigma\right)\left(u_{1}+\epsilon v_{1}\right)}{\epsilon+1}+ \\
\mu(1+\epsilon) \dot{v}_{2}+\alpha(1+\epsilon) v_{2}^{3}+ \\
\frac{\epsilon \varphi_{2}\left(u_{2}+\epsilon v_{2}\right)^{3}}{(\epsilon+1)^{3}}+\frac{\epsilon^{2} \varphi_{1}\left(\gamma_{b}+\sigma\right)\left(u_{1}+\epsilon v_{1}\right)^{3}}{(\epsilon+1)^{3}}=0 .
\end{gathered}
$$

Secondly, the complexification consists in introducing the following change of variable

$$
\begin{array}{ll}
\psi_{1}=\dot{u}_{1}+j \omega_{s t} u_{1}, & \psi_{2}=\dot{v}_{1}+j \omega_{s t} v_{1}, \\
\psi_{3}=\dot{u}_{2}+j \omega_{s t} u_{2}, & \psi_{4}=\dot{v}_{2}+j \omega_{s t} v_{2} .
\end{array}
$$

where $j^{2}=-1$ and $\omega_{s t}$ is the frequency for which the observed steady-state responses are assumed to oscillate. Here, the observation of the responses made in Sect. 4 lead us to state $\omega_{s t}=1$.

Then, the variable $u_{1}, v_{1}, u_{2}, v_{2}$ and their first and second derivatives with respect to time $t^{*}$ are expressed in term of the new variables $\psi_{i}$ (with $i \in[1,4]$ ) as:

$$
\begin{array}{ll}
u_{1}=\frac{\psi_{1}-\bar{\psi}_{1}}{2 j}, & u_{2}=\frac{\psi_{3}-\bar{\psi}_{3}}{2 j}, \\
\dot{u}_{1}=\frac{\psi_{1}+\bar{\psi}_{1}}{2}, & \dot{u}_{2}=\frac{\psi_{3}+\bar{\psi}_{3}}{2}, \\
\ddot{u}_{1}=\dot{\psi}_{1}-\frac{j}{2}\left(\psi_{1}+\bar{\psi}_{1}\right), & \ddot{u}_{2}=\dot{\psi}_{3}-\frac{j}{2}(19 \mathrm{~b}) \\
\left.v_{1}=\frac{\psi_{2}-\bar{\psi}_{2}}{2 j}, \bar{\psi}_{3}\right), & v_{2}=\frac{\psi_{4}-\bar{\psi}_{4}}{2 j}
\end{array}
$$




$$
\begin{array}{ll}
\dot{v}_{1}=\frac{\psi_{2}+\bar{\psi}_{2}}{2}, & \dot{v}_{2}=\frac{\psi_{4}+\bar{\psi}_{4}}{2}, \\
\ddot{v}_{1}=\dot{\psi}_{2}-\frac{j}{2}\left(\psi_{2}+\bar{\psi}_{2}\right), & \ddot{v}_{2}=\dot{\psi}_{4}-\frac{j}{2}\left(\psi_{4}+\bar{\psi}_{4}\right),
\end{array}
$$

where $\bar{\psi}_{i}$ is the complex conjugate of $\psi_{i}$.

Numerical results shown in Sect. 4 motivate us to assume that the variable $u_{1}, v_{1}, u_{2}$ and $v_{2}$ may be broken down into fast and slow components. For that, the following representation is introduced

$$
\begin{array}{ll}
\psi_{1}=\phi_{1} e^{j t^{*}}, & \psi_{2}=\phi_{2} e^{j t^{*}}, \\
\psi_{3}=\phi_{3} e^{j t^{*}}, & \psi_{4}=\phi_{4} e^{j t^{*}},
\end{array}
$$

where $\phi_{i}$ (with $i \in[1,4]$ ) is the complex slow modulated amplitude of the fast component $e^{j t^{*}}$.

Substituting Eqs. (19) into Eqs. (17) an equivalent complex system of differential equations is obtained. Then, using Eq. (20) in this complex system and performing an averaging over one period equal to $2 \pi$ yield to a system of equations describing the behavior of the slow complex amplitudes $\phi_{i}$. Finally, since $0<\epsilon \ll 1$, these equations are expanded in a first-order Taylor series around $\epsilon=0$ giving

$$
\begin{aligned}
\dot{\phi}_{1} & =-\frac{1}{2} j \epsilon\left(\phi_{1}\left(\frac{-3 \varphi_{1}\left|\phi_{1}\right|^{2}}{4}-j \eta_{1}+1\right)\right. \\
& \left.+\phi_{3}\left(\gamma_{b}+\sigma\right)-\phi_{2}\right) \\
\dot{\phi}_{2} & =\frac{1}{2} j\left(\frac{3 \alpha \phi_{2}\left|\phi_{2}\right|^{2}}{4}+\phi_{1}-\phi_{2}(1-j \mu)\right) \\
& +\frac{1}{2} \epsilon\left(\frac{3 j \alpha \phi_{2}\left|\phi_{2}\right|^{2}+3 j \phi_{1} \varphi_{1}\left|\phi_{1}\right|^{2}}{4}\right) \\
& \left.+\phi_{1}\left(\eta_{1}-j\right)-\phi_{2}(\mu-1)-j \phi_{3}\left(\gamma_{b}+\sigma\right)\right) \\
\dot{\phi}_{3} & =\frac{1}{2} j \epsilon\left(\frac{\phi_{3}\left(\frac{3 \varphi_{2}\left|\phi_{3}\right|^{2}}{4}+j \eta-1-2 a\right)}{4}\right) \\
& \left.+\phi_{1}\left(\gamma_{b}+\sigma\right)+\phi_{4}\right) \\
\dot{\phi}_{4} & =\frac{1}{2} j\left(\frac{3 \alpha \phi_{4}\left|\phi_{4}\right|^{2}}{4}+\phi_{3}-\phi_{4}(1-j \mu)\right) \\
& \frac{1}{2} j\left(\frac{3 \alpha \phi_{4}\left|\phi_{4}\right|^{2}+3 \phi_{3} \varphi_{2}\left|\phi_{3}\right|^{2}}{4}\right.
\end{aligned}
$$

$$
\left.+\phi_{1}\left(\gamma_{b}+\sigma\right)-\phi_{3}\left(1+2 a-j \eta_{2}\right)+\phi_{4}(1+j \mu)\right) \text {. }
$$

Eqs. (21) describe Complex Form of the Slow-Flow (CFSF) of the system (17) and it does not depend on the linear stiffness part of the NES.

The trivial fixed point is common to both the nonaveraged system (6) (or (17)) and the slow-flow (21). The stability of this trivial fixed point is calculated in Sect. 3 directly on the RHM+NES. On the other hand, the nontrivial fixed points of the slow-flow (21) (defined as $\dot{\phi}_{i}=0$ for $i \in[1,4]$ ) only characterizes periodic solutions of Eqs. (6) (or (17)) if the frequency of the periodic solutions is exactly equal to 1 , the frequency used to defined the complex variables (18). However, computing the real form of the slow-flow by using the polar coordinates $n_{i}(t)$ and $\theta_{i}(t)$ (with $i \in[1,4])$, defined by

$$
\phi_{i}(t)=n_{i}(t) e^{j \theta_{i}(t)}
$$

and considering not the arguments $\theta_{i}(t)$ directly but the argument differences $\delta_{2 i}=\theta_{2}(t)-\theta_{i}(t)$ (the master component can be chosen arbitrary, $\phi_{2}(t)$ is chosen for convenience), the periodic solutions of the system of Eqs. (21) (and consequently of the RHM+NES (6)) may be defined as the nontrivial fixed points of the system of the Real Form of the Slow-Flow (RFSF) which may be formally written as follows

$$
\dot{\mathbf{X}}=\mathbf{F}_{\mathbf{3}}(\mathbf{X}), \text { with } \mathbf{X}=\left[\begin{array}{lllll}
n_{1} & n_{2} n_{3} n_{4} & \delta_{21} & \delta_{23} & \delta_{24}
\end{array}\right]^{t}
$$

See Appendix A for more details about the link between the periodic solutions of the RHM+NES (on the form (17)) and the fixed points of the RFSF (23).

To summarize, the prediction of a situation in which a fixed points of the RFSF (23) is reached allows to predict the existence of stable periodic responses of RHM+NES. However, the numerical solution of $\mathbf{F}_{\mathbf{3}}(\mathbf{X})=\mathbf{0}$, i.e. the fixed points, cannot be accessed with a regular computer. Therefore, in the following section, an asymptotic analysis of the slow-flow is developed which permits to easily compute the fixed points and to analyze the responses regime when fixed points are unstable. 


\subsection{The Critical Manifold}

Slow and Super-slow subsystems. The slowflow described by Eqs. (21) can be written as follows:

$$
\begin{aligned}
& \dot{\phi}_{1}=\epsilon f_{1}\left(\phi_{1}, \phi_{2}, \phi_{3}, \phi_{4}\right) \\
& \dot{\phi}_{2}=f_{2}\left(\phi_{1}, \phi_{2}, \phi_{3}, \phi_{4}, \epsilon\right) \\
& \dot{\phi}_{3}=\epsilon f_{3}\left(\phi_{1}, \phi_{2}, \phi_{3}, \phi_{4}\right) \\
& \dot{\phi}_{4}=f_{4}\left(\phi_{1}, \phi_{2}, \phi_{3}, \phi_{4}, \epsilon\right),
\end{aligned}
$$

which highlights the "slow/fast" nature of the system. Here terminology introduced by Gendelman and Bar [2010] is preferred, i.e. the terms fast and slow are replaced by slow and super-slow respectively, whereby the term fast denotes the time scale determined by fast oscillations of the primary system with frequency 1. Therefore, system (24) consists of two super-slow complex variables $\phi_{1}$ and $\phi_{3}$ and two slow variables $\phi_{2}$ and $\phi_{4}$.

Eqs. (24) can be reformulated by switching from the slow time scale $t^{*}$ to the super-slow time scale $\tau=\epsilon t^{*}$ as

$$
\begin{aligned}
\Phi_{1}^{\prime} & =f_{1}\left(\Phi_{1}, \Phi_{2}, \Phi_{3}, \Phi_{4}\right) \\
\epsilon \Phi_{2}^{\prime} & =f_{2}\left(\Phi_{1}, \Phi_{2}, \Phi_{3}, \Phi_{4}, \epsilon\right) \\
\Phi_{3}^{\prime} & =f_{3}\left(\Phi_{1}, \Phi_{2}, \Phi_{3}, \Phi_{4}\right) \\
\epsilon \Phi_{4}^{\prime} & =f_{4}\left(\Phi_{1}, \Phi_{2}, \Phi_{3}, \Phi_{4}, \epsilon\right),
\end{aligned}
$$

where $^{\prime}=\frac{d}{d \tau}$ and $\Phi_{i}(\tau)=\phi_{i}\left(t=\frac{\tau}{\epsilon}\right)$. Solutions of the super-slow/slow system (24) (or (25)) can exhibit slow and super-slow epochs characterized by the speed at which the solution advances.

Stating $\epsilon=0$, the following subsystems are derived from (24) and (25) respectively:

$$
\begin{aligned}
& \dot{\phi}_{1}=0 \\
& \dot{\phi}_{2}=f_{2}\left(\phi_{1}, \phi_{2}, \phi_{3}, \phi_{4}, 0\right) \\
& \dot{\phi}_{3}=0 \\
& \dot{\phi}_{4}=f_{4}\left(\phi_{1}, \phi_{2}, \phi_{3}, \phi_{4}, 0\right),
\end{aligned}
$$

which is the slow subsystem, and

$$
\begin{aligned}
\Phi_{1}^{\prime} & =f_{1}\left(\Phi_{1}, \Phi_{2}, \Phi_{3}, \Phi_{4}\right) \\
0 & =f_{2}\left(\Phi_{1}, \Phi_{2}, \Phi_{3}, \Phi_{4}, 0\right) \\
\Phi_{3}^{\prime} & =f_{3}\left(\Phi_{1}, \Phi_{2}, \Phi_{3}, \Phi_{4}\right)
\end{aligned}
$$

$$
0=f_{4}\left(\Phi_{1}, \Phi_{2}, \Phi_{3}, \Phi_{4}, 0\right),
$$

which is the super-slow subsystem.

In the following sections the Geometric Singular Perturbation Theory (GSPT) is used to describe the dynamics of the full system (24) (and (25)) for $0<$ $\epsilon \ll 1$ from the analysis of the slow and superslow subsystems (26) and (27) (which are defined for $\epsilon=0)$. More precisely, the following result of the GSPT is used: if $0<\epsilon \ll 1$, the dynamics of the full system (24) (or (25)) during slow (resp. superslow) epoch is given by the dynamic of the slow (resp. super-slow) subsystem (26) (resp. (27)).

Stability of the Critical Manifold. The algebraic equations (27b) and (27d) of the super-slow subsystem define the so-called Critical Manifold (CM) (Jones [1995])

$$
\begin{aligned}
& C M:= \\
& \left\{\left(z_{1}, z_{2}, z_{3}, z_{4}\right) \in \mathbb{C}^{4} \mid\left\{f_{2}\left(z_{1}, z_{2}, z_{3}, z_{4}, 0\right)=0,\right.\right. \\
& \left.\left.\quad f_{4}\left(z_{1}, z_{2}, z_{3}, z_{4}, 0\right)=0\right\}\right\} .
\end{aligned}
$$

Looking at Eqs. (21b) and (21d) one see that the functions $f_{2}\left(\Phi_{1}, \Phi_{2}, \Phi_{3}, \Phi_{4}, 0\right)$ and $f_{4}\left(\Phi_{1}, \Phi_{2}, \Phi_{3}, \Phi_{4}, 0\right)$ have the same form. Therefore, from Eqs. (27b) and $(27 \mathrm{~d})$ the $\mathrm{CM}$ can take the following equivalent form

$$
\begin{aligned}
& \Phi_{1}(\tau)=\Phi_{2}(\tau) F\left(\left|\Phi_{2}(\tau)\right|\right) \\
& \Phi_{3}(\tau)=\Phi_{4}(\tau) F\left(\left|\Phi_{4}(\tau)\right|\right),
\end{aligned}
$$

where the complex function $F$ is defined by

$$
F(X)=F_{R}(X)+j F_{I}(X)=1-\frac{3 \alpha}{4} X^{2}-j \mu .
$$

Eq. (30) shows that the function $F$, which defines the CM, depends only on the NES parameters $\alpha$ and $\mu$. The fact that Eq. (29a) and (29b) are defined with the same function is the direct consequence of using two identical NES.

It is convenient to characterize the CM in $\mathbb{R}$. To achieve this, again polar coordinates are introduced 


$$
\Phi_{i}(\tau)=N_{i}(\tau) e^{j \Theta_{j}(\tau)} \text { for } i=1,2,3,4,
$$

and the module and the argument of (29) are successively computed, that lies to

$$
\begin{aligned}
N_{1}^{2} & =N_{2}^{2}\left[F_{R}\left(N_{2}\right)^{2}+F_{I}\left(N_{2}\right)^{2}\right]=H\left(N_{2}\right) \\
\Theta_{1} & =\Theta_{2}+\arg \left(F\left(N_{2}\right)\right) \\
N_{3}^{2} & =N_{4}^{2}\left[F_{R}\left(N_{4}\right)^{2}+F_{I}\left(N_{4}\right)^{2}\right]=H\left(N_{4}\right) \\
\Theta_{3} & =\Theta_{4}+\arg \left(F\left(N_{4}\right)\right) .
\end{aligned}
$$

The local extrema of the real function $H(X)$ are given by the positive roots of its derivative $H^{\prime}(X)$. An easy calculus shows that the local extrema occur at

$$
\begin{aligned}
& X_{M}=\frac{2}{3} \sqrt{\frac{2-\sqrt{1-3 \mu^{2}}}{\alpha}} \\
& X_{m}=\frac{2}{3} \sqrt{\frac{2+\sqrt{1-3 \mu^{2}}}{\alpha}}
\end{aligned}
$$

if the following relation holds

$$
\mu<\frac{1}{\sqrt{3}},
$$

and in this case $X_{M}<X_{m}$. The chosen value of $\mu$ (see Eq. (10)) satisfies the previous relation.

Because $\dot{\phi}_{2}$ and $\dot{\phi}_{4}$ in (26) are reduced to zero in the $\mathrm{CM}$, each point of the $\mathrm{CM}$ is a fixed point for the slow subsystem which consists in two independent systems of equations

$$
\begin{aligned}
& \dot{\phi}_{1}=0 \\
& \dot{\phi}_{2}=\frac{j}{2}\left[\phi_{1}-\phi_{2} F\left(\left|\phi_{2}\right|\right)\right],
\end{aligned}
$$

and,

$$
\begin{aligned}
& \dot{\phi}_{3}=0 \\
& \dot{\phi}_{4}=\frac{j}{2}\left[\phi_{3}-\phi_{4} F\left(\left|\phi_{4}\right|\right)\right] .
\end{aligned}
$$

To determine the stability of the $\mathrm{CM}$, one must know if the CM attracts or repels the slow dynamics. For that, using the polar coordinates $\phi_{i}=n_{i} e^{j \theta_{i}}$, Eqs. (36) and (37) are rewritten as follows

$$
\begin{aligned}
\dot{n}_{1} & =0 \\
\dot{n}_{2} & =\frac{1}{2}\left(n_{1} \sin \delta_{21}+n_{2} F_{I}\left(n_{2}\right)\right) \\
\dot{\delta}_{21} & =\frac{1}{2}\left(\frac{n_{1}}{n_{2}} \cos \delta_{21}-F_{R}\left(n_{2}\right)\right),
\end{aligned}
$$

and,

$$
\begin{aligned}
\dot{n}_{3} & =0 \\
\dot{n}_{4} & =\frac{1}{2}\left(n_{3} \sin \delta_{43}+n_{2} F_{I}\left(n_{4}\right)\right) \\
\dot{\delta}_{43} & =\frac{1}{2}\left(\frac{n_{3}}{n_{4}} \cos \delta_{43}-F_{R}\left(n_{4}\right)\right)
\end{aligned}
$$

where the argument differences $\delta_{21}=\theta_{2}-\theta_{1}$ and $\delta_{43}=\theta_{4}-\theta_{3}$ have been introduced. Stability range of the CM is then determined by examining the sign of the eigenvalues real parts of the Jacobian matrix of the two identical differential systems (38) and (39) on the CM. It can be shown that the condition of stability of the $\mathrm{CM}$ is equivalent to

$$
H^{\prime}(X)>0,
$$

and the stability range of the $\mathrm{CM}$ is characterized by the points $\left(X_{m}, Y_{m}\right)$ and $\left(X_{M}, Y_{M}\right)$ where $Y_{m}=$ $\sqrt{H\left(X_{m}\right)}$ and $Y_{M}=\sqrt{H\left(X_{M}\right)}$. Such points on which the CM ceases to be hyperbolic ${ }^{3}$ connecting attractive and repulsive parts of the the $\mathrm{CM}$ are called folded singularities. We point that the folded singularities exist whatever the values of the argument differences $\delta_{21}=\theta_{2}-\theta_{1}$ and $\delta_{43}=\theta_{4}-\theta_{3}$.

A typical Critical Manifold and its stability range are depicted (see Fig. 6) in which $X_{d}$ and $X_{u}$ are solutions of

$H\left(X_{m}\right)=H\left(X_{d}\right) \Rightarrow X_{d}=\frac{2 \sqrt{2}}{3} \sqrt{\frac{1-\sqrt{1-3 \mu^{2}}}{\alpha}}$,

and,

$H\left(X_{M}\right)=H\left(X_{u}\right) \Rightarrow X_{u}=\frac{2 \sqrt{2}}{3} \sqrt{\frac{1+\sqrt{1-3 \mu^{2}}}{\alpha}}$,

\footnotetext{
${ }^{3}$ The CM is hyperbolic if all eigenvalues of the Jacobian matrices of the differential systems (38) and (39) have nonzero real part.
} 


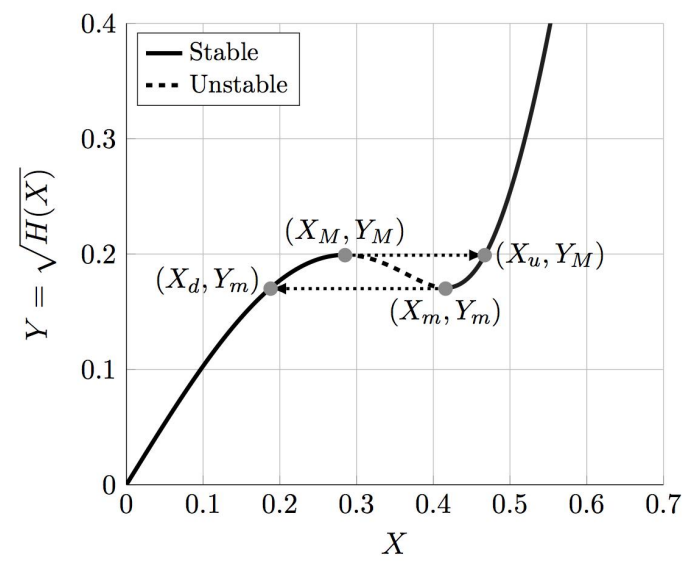

Figure 6: Critical Manifold (CM). Following parameters are used: $\alpha=7$ and $\mu=0.4$.

respectively.

\subsection{Fixed points and folded singularities of the slow-flow}

Introducing the CM Eq. (29) in Eqs. (27a) and (27c), the super-slow subsystem is written only with respect to the variables $\Phi_{2}$ and $\Phi_{4}$

$\frac{\partial\left[\Phi_{2} F\left(\left|\Phi_{2}\right|\right)\right]}{\partial \tau}=f_{1}\left(\Phi_{2} F\left(\left|\Phi_{2}\right|\right), \Phi_{2}, \Phi_{4} F\left(\left|\Phi_{4}\right|\right), \Phi_{4}\right)$

$\frac{\partial\left[\Phi_{4} F\left(\left|\Phi_{4}\right|\right)\right]}{\partial \tau}=f_{3}\left(\Phi_{2} F\left(\left|\Phi_{2}\right|\right), \Phi_{2}, \Phi_{4} F\left(\left|\Phi_{4}\right|\right), \Phi_{4}\right)$.

Using the polar coordinates (31) and separating real and imaginary parts, Eqs. (43) take the following forms

$$
\begin{array}{r}
F_{R}\left(N_{2}\right) \frac{\partial N_{2}}{\partial \tau}-N_{2} \frac{\partial \Theta_{2}}{\partial \tau} F_{I}\left(N_{2}\right)+N_{2} \frac{\partial N_{2}}{\partial \tau} F_{R}^{\prime}\left(N_{2}\right) \\
=f_{1, R}\left(N_{2}, N_{4}, \Delta_{24}\right) \\
(44 \mathrm{a}) \\
N_{2} \frac{\partial \Theta_{2}}{\partial \tau} F_{R}\left(N_{2}\right)+\frac{\partial N_{2}}{\partial \tau} F_{I}\left(N_{2}\right)+N_{2} \frac{\partial N_{2}}{\partial \tau} F_{I}^{\prime}\left(N_{2}\right) \\
=f_{1, I}\left(N_{2}, N_{4}, \Delta_{24}\right)
\end{array}
$$

$$
\begin{array}{r}
F_{R}\left(N_{4}\right) \frac{\partial N_{4}}{\partial \tau}-N_{4} \frac{\partial \Theta_{4}}{\partial \tau} F_{I}\left(N_{4}\right)+N_{4} \frac{\partial N_{4}}{\partial \tau} F_{R}^{\prime}\left(N_{4}\right) \\
=f_{3, R}\left(N_{2}, N_{4}, \Delta_{24}\right)
\end{array}
$$

$$
\begin{array}{r}
N_{4} \frac{\partial \Theta_{4}}{\partial \tau} F_{R}\left(N_{4}\right)+\frac{\partial N_{4}}{\partial \tau} F_{I}\left(N_{4}\right)+N_{4} \frac{\partial N_{4}}{\partial \tau} F_{I}^{\prime}\left(N_{4}\right) \\
=f_{3, I}\left(N_{2}, N_{4}, \Delta_{24}\right)
\end{array}
$$

where the expressions of the functions $f_{1, R}, f_{1, I}, f_{3, R}$ and $f_{3, I}$ are respectively given by

$$
\begin{gathered}
f_{1, R}\left(N_{2}, N_{4}, \Delta_{24}\right)=\operatorname{Re}\{ \\
f_{1}\left(N_{2} e^{j \Theta_{2}} F\left(N_{2}\right), N_{2} e^{j \Theta_{2}}, N_{4} e^{j \Theta_{4}} F\left(N_{4}\right), N_{4} e^{j \Theta_{4}}\right) \\
\left.\times e^{-j \Theta_{2}}\right\}, \quad(45)
\end{gathered}
$$

$$
\begin{gathered}
f_{1, I}\left(N_{2}, N_{4}, \Delta_{24}\right)=\operatorname{Im}\{ \\
f_{1}\left(N_{2} e^{j \Theta_{2}} F\left(N_{2}\right), N_{2} e^{j \Theta_{2}}, N_{4} e^{j \Theta_{4}} F\left(N_{4}\right), N_{4} e^{j \Theta_{4}}\right) \\
\left.\times e^{-j \Theta_{2}}\right\}, \quad(46)
\end{gathered}
$$

$$
\begin{gathered}
f_{3, R}\left(N_{2}, N_{4}, \Delta_{24}\right)=\operatorname{Re}\{ \\
f_{3}\left(N_{2} e^{j \Theta_{2}} F\left(N_{2}\right), N_{2} e^{j \Theta_{2}}, N_{4} e^{j \Theta_{4}} F\left(N_{4}\right), N_{4} e^{j \Theta_{4}}\right) \\
\left.\times e^{-j \Theta_{4}}\right\}, \quad(47
\end{gathered}
$$

$$
\begin{gathered}
f_{3, I}\left(N_{2}, N_{4}, \Delta_{24}\right)=\operatorname{Im}\{ \\
f_{3}\left(N_{2} e^{j \Theta_{2}} F\left(N_{2}\right), N_{2} e^{j \Theta_{2}}, N_{4} e^{j \Theta_{4}} F\left(N_{4}\right), N_{4} e^{j \Theta_{4}}\right) \\
\left.\times e^{-j \Theta_{4}}\right\}, \quad(48
\end{gathered}
$$

involving the argument difference $\Delta_{24}=\Theta_{2}-\Theta_{4}$. Combining Eqs. (44a-44d), system of Eqs. (44) can be reduced to the following forms

$$
\begin{aligned}
& g\left(N_{2}\right) \frac{\partial N_{2}}{\partial \tau}=f_{N_{2}}\left(N_{2}, N_{4}, \Delta_{24}\right) \\
& g\left(N_{2}\right) \frac{\partial \Theta_{2}}{\partial \tau}=f_{\Theta_{2}}\left(N_{2}, N_{4}, \Delta_{24}\right) \\
& g\left(N_{4}\right) \frac{\partial N_{4}}{\partial \tau}=f_{N_{4}}\left(N_{2}, N_{4}, \Delta_{24}\right) \\
& g\left(N_{4}\right) \frac{\partial \Theta_{4}}{\partial \tau}=f_{\Theta_{4}}\left(N_{2}, N_{4}, \Delta_{24}\right),
\end{aligned}
$$

and finally

$$
g\left(N_{2}\right) \frac{\partial N_{2}}{\partial \tau}=f_{N_{2}}\left(N_{2}, N_{4}, \Delta_{24}\right)
$$




$$
\begin{aligned}
g\left(N_{4}\right) \frac{\partial N_{4}}{\partial \tau} & =f_{N_{4}}\left(N_{2}, N_{4}, \Delta_{24}\right) \\
g\left(N_{2}\right) g\left(N_{4}\right) \frac{\partial \Delta_{24}}{\partial \tau} & =f_{\Delta_{24}}\left(N_{2}, N_{4}, \Delta_{24}\right),
\end{aligned}
$$

where

$$
\begin{aligned}
& f_{\Delta_{24}}\left(N_{2}, N_{4}, \Delta_{24}\right)= \\
& g\left(N_{4}\right) f_{\Theta_{2}}\left(N_{2}, N_{4}, \Delta_{24}\right)-g\left(N_{2}\right) f_{\Theta_{4}}\left(N_{2}, N_{4}, \Delta_{24}\right),
\end{aligned}
$$

and

$$
g(X)=\frac{H^{\prime}(X)}{2} .
$$

From Eqs. (50), it is possible to detect fixed points and folded singularities.

Fixed points. The (regular) fixed points of Eqs. (50), $\left\{N_{2}^{e}, N_{4}^{e}, \Delta_{24}^{e}\right\}$, are defined by

$$
\begin{aligned}
f_{N_{2}}\left(N_{2}^{e}, N_{4}^{e}, \Delta_{24}^{e}\right) & =0 \\
f_{N_{4}}\left(N_{2}^{e}, N_{4}^{e}, \Delta_{24}^{e}\right) & =0 \\
f_{\Delta_{24}}\left(N_{2}^{e}, N_{4}^{e}, \Delta_{24}^{e}\right) & =0 \\
g\left(N_{2}^{e}\right) & \neq 0 \\
g\left(N_{4}^{e}\right) & \neq 0 .
\end{aligned}
$$

Since $0<\epsilon \ll 1$, fixed points computed from Eqs. (53) corresponds to fixed points of the real RFSF (23). The expression of these fixed points are obtained from Eq. (32)

$$
\begin{aligned}
n_{1}^{e} & =\sqrt{H\left(N_{2}^{e}\right)} \\
n_{2}^{e} & =N_{2}^{e} \\
n_{3}^{e} & =\sqrt{H\left(N_{4}^{e}\right)} \\
n_{4}^{e} & =N_{4}^{e} \\
\delta_{21}^{e} & =-\arg \left(F\left(N_{2}\right)\right) \\
\delta_{23}^{e} & =\Delta_{24}^{e}-\arg \left(F\left(N_{4}\right)\right) \\
\delta_{24}^{e} & =\Delta_{24}^{e} .
\end{aligned}
$$

Stability of the fixed points can now be found by looking the sign of the eigenvalues real parts of the Jacobian matrix of the vector function $\mathbf{F}_{\mathbf{3}}$ (see Eq. (23)) evaluated at

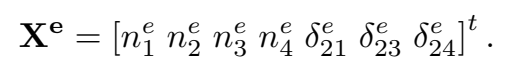

Folded singularities. The system of Eqs. (50) is not defined on the folded singularities defined in Sect. 5.2 because the system is singular on these points. Introducing, the desingularized super-slow subsystem, which is obtained using the time rescaling $\tau \rightarrow \tau^{\prime} g\left(N_{2}\right) g\left(N_{4}\right)$

$$
\begin{aligned}
\frac{\partial N_{2}}{\partial \tau^{\prime}} & =g\left(N_{4}\right) f_{N_{2}}\left(N_{2}, N_{4}, \Delta_{24}\right) \\
\frac{\partial N_{4}}{\partial \tau^{\prime}} & =g\left(N_{2}\right) f_{N_{4}}\left(N_{2}, N_{4}, \Delta_{24}\right) \\
\frac{\partial \Delta_{24}}{\partial \tau^{\prime}} & =f_{\Delta_{24}}\left(N_{2}, N_{4}, \Delta_{24}\right),
\end{aligned}
$$

one can notice that the folded singularities (here denoted $\left.\left\{N_{2}^{s}, N_{4}^{s}, \Delta_{24}^{s}\right\}\right)$ can be also defined as the fixed points of the desingularized super-slow subsystem (56) (see for example Desroches et al. [2012]). Since $g\left(X_{m / M}\right)=0$ (where $X_{m / M}$ denotes indistinctly $X_{M}$ or $\left.X_{m}\right)$ and looking at the definition of the function $f_{\Delta_{24}}$ (see Eq. 51), folded singularities are defined from (56) by

$$
\left\{N_{2}^{s}, N_{4}^{s}, \Delta_{24}^{s}\right\}=\left\{X_{m / M}, X_{m / M}\right\}, \forall \Delta_{24} .
$$

\section{Exploitation of theoretical results and discussion}

\subsection{Explanation of the observed responses}

The existence of an S-shaped CM (i.e. the presence of folded singularities on which the stability of the CM changes) allows to explain the three steady-state regimes of the RHM+NES (6) observed when its trivial solution is unstable, namely: Mitigation through Periodic Response, Mitigation through Strongly Modulated Response (SMR) and No mitigation.

A fixed point of the RedM is reached. These situations corresponds to a periodic solutions of the RHM+NES which may correspond to either mitigation through Periodic Response or No mitigation of the instability (see Sect. 4). Indeed, the bifurcation diagram of the super-slow subsystem (50) with respect the variables $N_{1}$ (see Fig. 7(a)) and $N_{3}$ (see Fig. 7(b)) highlights two ranges of $\sigma$ in which stable fixed points are encountered (see Fig. 7(a)):

- Domain 1: $0.31 \lesssim \sigma \lesssim 0.72$,

- Domain 2: $\sigma \gtrsim 0.93$. 


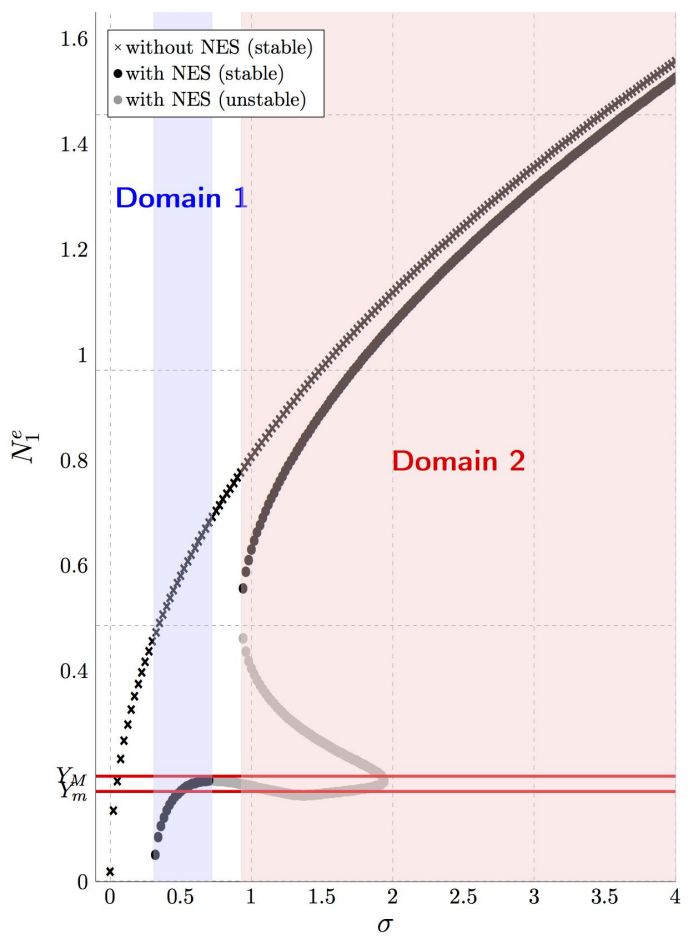

(a)

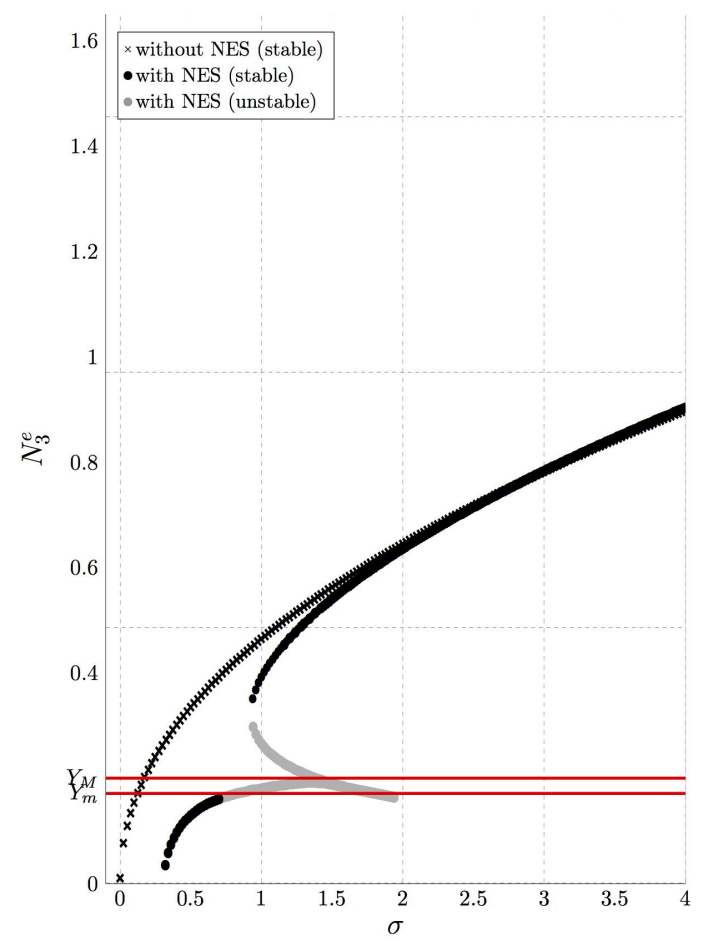

(b)

Figure 7: Comparison between the bifurcation diagrams of the slow-flow of the system without NES (RPS (7)) and with NES which is obtained from the analysis of the super-slow subsystem (50); with respect to the variable $N_{1}$ (a) and to the variable $N_{3}$ (b). Set of parameters (10) is used.

The comparison with the bifurcation diagram of the RPS $(7)^{4}$ (see also Fig. 7) allows to obtain to following conclusion: if a stable fixed point is reached in the domain 1, mitigation through Periodic Response are observed and if a stable fixed point is reached in the domain 2 , this time, no suppression of the instability is observed.

Relaxation oscillations. The S-shape of the CM suggests also the possible existence of relaxation oscillations (Grasman [1987]): after reaching of fold singularity $\left(X_{M}, Y_{M}\right)$ (in the $\left(N_{2}, N_{1}\right)$-plane or in the $\left(N_{4}, N_{3}\right)$-plane), the system jumps to $\left(X_{u}, Y_{M}\right)$, which is followed by a super-slow evolution of the trajectory of the system (in the stable domain of the CM) until it reaches $\left(X_{m}, Y_{m}\right)$. After another jump and a super-slow evolution (the stable domain of the $\mathrm{CM}$ ), the trajectory returns to $\left(X_{M}, Y_{M}\right)$ (see Fig. 6). Such scenario of relaxation oscillations for the slow-flow can explain the existence of Strongly Modulated Responses

\footnotetext{
${ }^{4}$ The bifurcation diagram of the RPS (7) is also obtained computing the stability of the non trivial fixed points of its slow-flow expanded in fist-order Taylor series around $\epsilon=0$.
}

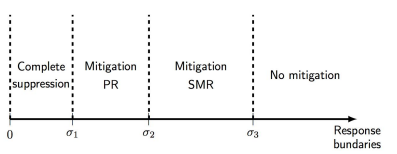

Figure 8: Outline schematic showing the definition of the boundary values $\sigma_{1}, \sigma_{2}$ and $\sigma_{3}$ which separate the regions of existence of the four steady-state response regimes.

(Gendelman et al. [2010], Gendelman and Bar [2010], Starosvetsky and Gendelman [2008]) (SMR) for the RefM. Note that if $\mu>1 / \sqrt{3}$, the S-shape nature of the $\mathrm{CM}$ is lost and therefore relaxation oscillations are not possible.

\subsection{Prediction of the steady-state response regimes and limitation of the local linear stability analysis of the slow-flow}

The aim of this section is to predict, for a given set of parameters, the nature of the resulting steady-state response regimes of the RHM+NES (6). The prediction is performed checking first the local stability property of the trivial equilibrium point (see Sect. 3), this allowed to predict the appearance of Complete 


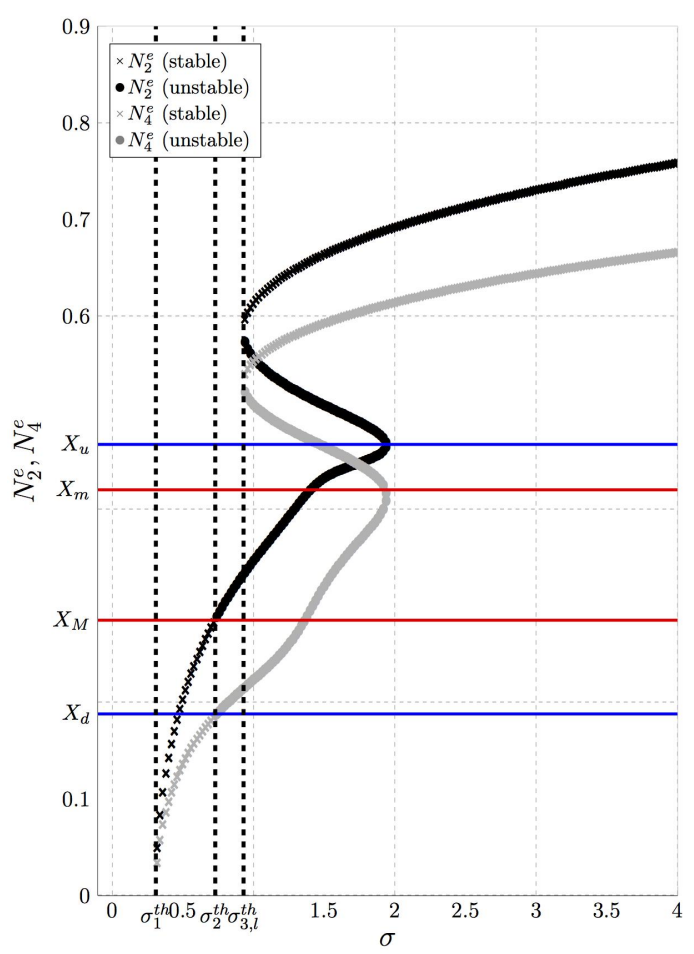

(a)

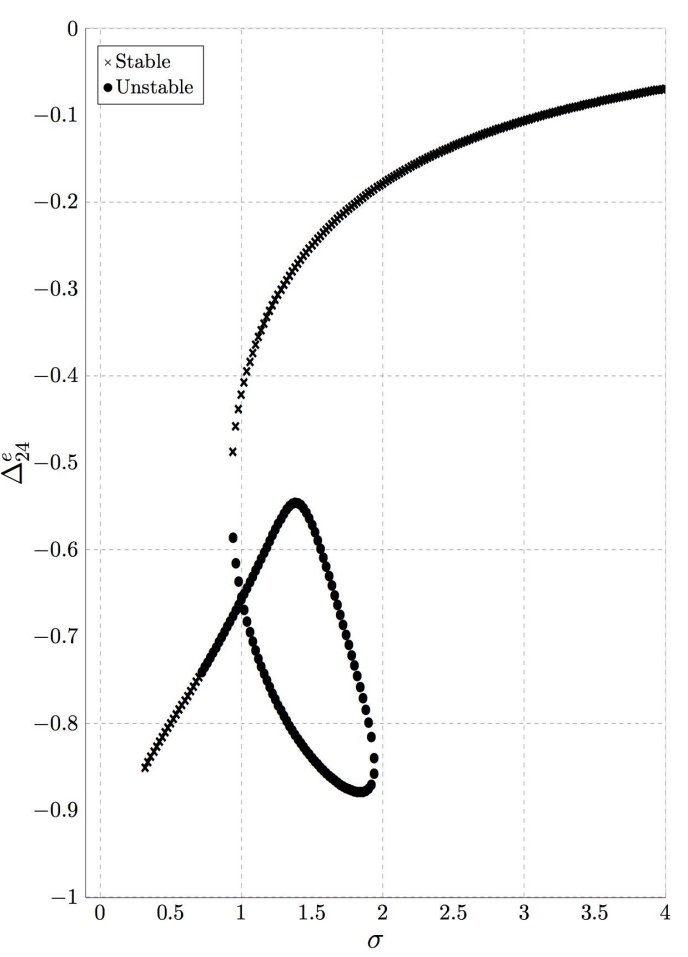

(b)

Figure 9: Bifurcation diagram of the slow-flow of the system with NES obtained from the analysis of the super-slow subsystem (50); with respect to the variable $N_{2}$ and $N_{4}$ (a) and to the variable $\Delta_{24}$ (b). Set of parameters (10) is used.

suppression of the instability. Then, if the trivial solution is unstable, we intent to discriminate the three other steady-state response regimes analyzing the local stability of the fixed points of the slow-flow (see Sect. 5).

To illustrate the prediction method and highlight its limitation, the study is restricted to a one parameter dependent system, namely the detuning parameter $\sigma$. To simplify the presentation of the method (but without limiting its generality), the set of parameters (10) has been chosen to allow a continuous evolution of the nature of the steady-state response regimes when the parameter $\sigma$ increases (see Fig. 8). In this case, the goal is to find the values $\sigma_{1}, \sigma_{2}$ and $\sigma_{3}$ which separate the regions of existence of the four steady-state response regimes.

From the simple local stability analysis of the trivial equilibrium performed in Sect. 3 , it is possible to obtain a theoretical value of $\sigma_{1}$ (denoted $\sigma_{1}^{t h}$ ), this is the difference between the Hopf bifurcation point of the system without and with NES (see Eq. (12)): $\sigma_{1}^{t h}=0.31$.

Fig. 9 shows the bifurcation diagram deduced from local stability analysis of the super-slow subsystem (50) performed in Sect. 5 (Fig. 9(a) with respect to the variables $N_{2}$ et $N_{4}$ and Fig. 9 (b) with respect to the argument difference $\Delta_{24}$ ). From this analysis, a theoretical value $\sigma_{2}^{t h}$ of $\sigma_{2}$ and a lower $\sigma_{3, l}^{t h}$ theoretical values of $\sigma_{3}$ can be deduced. Here, lower values mean that it is sure to obtain SMR mechanism if $\sigma<\sigma_{3, l}^{t h}$. However, if $\sigma>\sigma_{3, l}^{t h}$, it is not possible to conclude if SMR or no mitigation mechanisms are encountered. The theoretical value $\sigma_{2}^{\text {th }}$ is obtained as the first intersection between the bifurcation diagram of the super-slow subsystem (50) with respect the variables $N_{2}$ or $N_{4}$ and the line $N_{2}=N_{4}=X_{M}$. In Fig. 9(a), one can notice that the bifurcation diagram with respect to the variables $N_{2}$ is the first to cross the line $N_{2}=N_{4}=X_{M}$ at $\sigma=0.73$ which is the theoretical value of $\sigma_{2}^{t h}$. At this point, relaxation oscillations of the slow-flow are observed. Before the appearance of the second branch of stable fixed points at $\sigma \approx 0.93$, relaxation oscillations phenomenon is the only possible regime, this defines the lower theoretical value $\sigma_{3, l}^{t h}$.

The fact that only a lower theoretical value of 
the $\sigma_{3}$ can be obtained highlights the limitation of the method to predict unequivocally all the steadystate response regimes, this is due to the dimensionality of the super-slow flow subsystem. Indeed, in works in which the super-slow flow subsystem can be reduced to a one-dimensional system (Domany and Gendelman [2013], Gendelman et al. [2010], Gendelman and Bar [2010]), global structure of possible response regimes can be deduced directly from the local stability analysis of the slow-flow. Here the super-slow subsystem is a three-dimensional system. In this case, determination of the basin of attraction of each solution (fixed points and relaxation oscillations) would be needed to conclude about the nature of the steadystate regimes, this may be subject of future work.

\section{Additional analysis using numerical simulations and benchmark of theo- retical results}

In this section numerical simulations are first performed in order to explore more accurately the possible steady-state response regimes. Secondly, these numerical simulations allow to study the validity of the theoretical results presented in Sect. 6.2.

Figs. 10 to 14 present the same type of numerical simulations obtained using the set of parameters (10) and for five different values of $\sigma$. In each figure, it is depicted:

- Figs. (a), (b), (c) and (d) compare numerical simulations of the RHM+NES written using barycentric coordinates (see Eq. (17)) and numerical simulations of the slow-flow (21).

- Figs. (e) and (f) compare the Critical Manifold $\left(N_{1}=\sqrt{H\left(N_{2}\right)}\right.$ and $\left(N_{3}=\sqrt{H\left(N_{4}\right)}\right.$, see Eq. (32)) with numerical simulations of the slowflow.

- Figs. (g) and (h) compare the bifurcation diagram obtained from Eq. (53) with the trajectory of the slow-flow in the $3 \mathrm{D} N_{2}, N_{4}, \Delta_{24}$-space. Two views are used to make the reading of the $3 \mathrm{D}$ graph easier.

In Fig. 10 the chosen value of $\sigma=0.2$ is smaller than $\sigma_{1}^{t h}=0.31$ and Complete suppression is actually observed. In Fig. 11 the chosen value of $\sigma=0.6$ is between $\sigma_{1}^{t h}=0.31$ and $\sigma_{2}^{t h}=0.73$ and Mitigation through Periodic Response is actually observed. In
Fig. 12 the chosen value of $\sigma=0.9$ is between $\sigma_{2}^{t h}=$ 0.73 and $\sigma_{3, l}^{t h}=0.93$ and Mitigation through Strongly modulated Modulated Response is actually observed. We can notice in this example that relaxation oscillations of the slow-flow which explain the strongly modulated response of the RHM+NES are observed only in the $N_{1}, N_{2}$-plane and only small oscillation are observed in the $N_{3}, N_{4}$-plane (see Figs. $12(\mathrm{e})$ and $12(\mathrm{f})$ ). We call this SMR regime: semi-SMR regime. The value of $\sigma$ must be larger to observe relaxation oscillations in both $N_{1}, N_{2}$-plane and $N_{3}, N_{4}$-plane (see Fig. 13 obtained using $\sigma=1.3$ ). We call this SMR regime: full-SMR regime. Finally, in Fig. 14, the results are obtained with $\sigma=2$ and No mitigation is observed.

To observe more precisely the boundaries between the different observed regimes, the amplitudes of the steady-state responses of the numerical simulations of the RHM+NES written using barycentric coordinates (see Eq. (17)) and numerical simulations of the slowflow (21) are plotted and compared to the theoretical bifurcation diagram in Fig. 15. Following conclusion can be made from the observation of these figures:

Comparison between theoretical and numerical boundaries values of $\sigma$. The better figure to perform this comparison is Fig. 15(b). We can observe that the transition from Complete suppression to Mitigation through Periodic Response and the transition from Mitigation through Periodic Response to Mitigation through Strongly Modulated Response is well predicted by $\sigma_{1}^{t h}=0.31$ and $\sigma_{2}^{t h}=0.73$ respectively. Indeed, one can see that the steady-state amplitudes of the numerical simulations (for both RHM+NES and the slow-flow) follow the amplitudes predicted by the bifurcation diagram until $\sigma=\sigma_{2}^{t h}$. At this value a jump of the steady-state amplitudes of the numerical simulations is observed corresponding to the transition from periodic regimes to SMR.

Not predicted boundaries values of $\sigma$. The transition from Mitigation through Strongly Modulated Response to No mitigation and from semi-SMR regime to full-SMR regime have not theoretical values. However, they can be found on numerical simulations. Observing for example Fig. 15(b), one can see that the transition from Mitigation through Strongly Modulated Response to No mitigation of the dynamic instability appears at $\sigma=1.63$ (denoted $\sigma_{3}^{\text {num }}$ on the graph). The transition from semi-SMR regime to full- 
SMR regime is observed on Fig. 15(d), it appears at $\sigma=1.08$.

Prediction of the steady-state amplitudes The theoretical bifurcation diagram is obtained from the super-slow subsystem (see Eq.(53)). We can first see the good agreement between the theoretical bifurcation diagram and the steady-state amplitudes measures on numerical simulation of the slow-slow when the steady-state regime is a periodic regime, i.e. when $\sigma<\sigma_{2}^{\text {th }}$ and $\sigma>\sigma_{3}^{\text {num }}$. Moreover the theoretical values $\sqrt{H\left(X_{u}\right)}=Y_{M}$ and $X_{u}$ (see Fig. 6) give an approximated prediction of maximum amplitudes when full-SMR occurs, i.e. for $1.08<\sigma<\sigma_{3}^{\text {num }}$.

Comparison between RHM+NES and the slow-flow. The comparison between numerical simulations of RHM+NES and the slow-flow is important because the capacity of the slow-flow to reproduce the behavior of the RHM+NES reflect the quality of all mathematical developments which derive from (even those not presented in this paper). Observing Fig. 15, the comparison shows that the slow-flow well predicts the boundary values of $\sigma$. The slow-flow well predicts also the steady-state amplitudes except for periodic regimes in domain 2 (see Fig. 7) corresponding to $N_{o}$ mitigation of the dynamic instability. Indeed, one can observe a significant underestimation of the steadystate amplitude (here especially for the variable $N_{2}$, see Fig. 15(b)). However, in the context of the control of the instability the most pertinent values to predict are the boundaries, in particular the boundary $\sigma_{3}$ corresponding to the transition from Mitigation through Strongly Modulated Response to No mitigation of the instability. The fact that the slow-slow can describe this transition motive us to continue this research in order to find a theoretical value of $\sigma_{3}$.

\section{Conclusion}

We studied the capacity of Nonlinear Energy Sinks (NES) to mitigate vibrations due to mode-coupling instability in braking systems. To achieve that, a simple two degree of freedom model which can reproduce mode-coupling instability (i.e. the well-known Hultèn's model) was coupled to two ungrounded NES.

To analyze the steady-state response regimes, the system is partitioned in slow-fast dynamics using complexification-averaging approach. The presence a small dimensionless parameter related to the mass of the NES in the slow-flow system implies that it involves two "slow" complex variables and two "superslow" complex variables. The "super-slow/slow" nature of the system allowed us to use multiple scale approach to analyze it. In particular, the Critical Manifold of the slow-flow was determined. Its S-shape (i.e. involving two folded singularities) and the associated stability properties provide an analytical tool to explain the existence of three regimes: periodic response regimes, strongly modulated responses regimes and no mitigation regimes that appear when the trivial solution is unstable. A complete suppression regimes is also observed and it is studied directly on the full system.

The boundary values of the friction coefficient corresponding to the transition from complete suppression regime to periodic response regimes and from periodic response regimes to strongly modulated responses are predicted analytically. However the prediction of the boundary value between strongly modulated responses and no mitigation responses is not performed, this highlights that global structure of possible response regimes can not be deduced from local stability analysis of a super-slow flow subsystem with dimension larger than one. The prediction of this boundary value could be important in the context of engineering applications. That is why advanced mathematical procedure will be developed to predict this boundary.

\section{A Link between the periodic solutions of the RHM+NES and the fixed points of the RFSF}

To explain the link between the periodic solutions of the RHM+NES (on the form of (17)) and the fixed points of the RFSF (23), let's consider a periodic steady-state regime of the system (17) defined by

$$
\begin{aligned}
& u_{1}(t)=U_{1} \sin \left((1+e) t+\theta_{u_{1}}\right) \\
& v_{1}(t)=V_{1} \sin \left((1+e) t+\theta_{v_{1}}\right) \\
& u_{2}(t)=U_{2} \sin \left((1+e) t+\theta_{u_{2}}\right) \\
& v_{2}(t)=V_{2} \sin \left((1+e) t+\theta_{v_{2}}\right)
\end{aligned}
$$

where $U_{1}, V_{1}, U_{2}, V_{2}, \theta_{u_{1}}, \theta_{v_{1}}, \theta_{u_{2}}, \theta_{v_{2}}$ and $e$ are real constant. $e$ characterizes the error made using 1 as fast component frequency in the complexificationaveraging method with the assumption that $e \ll 1$. 
Using Eqs. (18) and (20), the steady-state regime described by (58) is written in terms of complex slow modulated amplitude $\phi_{i}$ (with $i \in[1,4]$ )

$$
\begin{aligned}
\phi_{1} & =\left(\dot{u}_{1}+j u_{1}\right) e^{-j t} \\
& =U_{1}\left(1+\frac{e}{2}\right) e^{j\left(e t+\theta_{u_{1}}\right)}+\text { t.h.f, } \\
\phi_{2} & =\left(\dot{v}_{1}+j v_{1}\right) e^{-j t} \\
& =V_{1}\left(1+\frac{e}{2}\right) e^{j\left(e t+\theta_{v_{1}}\right)}+\text { t.h.f, } \\
\phi_{3} & =\left(\dot{u}_{2}+j u_{2}\right) e^{-j t} \\
& =U_{2}\left(1+\frac{e}{2}\right) e^{j\left(e t+\theta_{u_{2}}\right)}+\text { t.h.f, } \\
\phi_{4} & =\left(\dot{v}_{2}+j v_{2}\right) e^{-j t} \\
& =V_{2}\left(1+\frac{e}{2}\right) e^{j\left(e t+\theta_{v_{2}}\right)}+\text { t.h.f, }
\end{aligned}
$$

where "t.h.f" means term of higher frequency.

Using the polar coordinates $n_{i}(t)$ and $\theta_{i}(t)$ (with $i \in[1,4])$ defined by Eq. (22), Eqs. (59) become

$$
\begin{aligned}
& n_{1}=U_{1}\left(1+\frac{e}{2}\right) \approx U_{1} \\
& n_{2}=V_{1}\left(1+\frac{e}{2}\right) \approx V_{1} \\
& n_{3}=U_{2}\left(1+\frac{e}{2}\right) \approx U_{2} \\
& n_{4}=V_{2}\left(1+\frac{e}{2}\right) \approx V_{2} \\
& \delta_{21}=\theta_{2}-\theta_{1}=\theta_{v_{1}}-\theta_{u_{1}} \\
& \delta_{23}=\theta_{2}-\theta_{3}=\theta_{v_{1}}-\theta_{u_{2}} \\
& \delta_{24}=\theta_{2}-\theta_{4}=\theta_{v_{1}}-\theta_{v_{2}} .
\end{aligned}
$$

Comparing Eqs. (59) and (60) we understand why the argument differences must be used. Indeed, even if $e \ll 1$, the error $e$ caused a linear growth of the arguments of the complex amplitude $\phi_{i}$, which are therefore not stationary.

\section{References}

Zahra Nili Ahmadabadi and Siamak E Khadem. Annihilation of high-amplitude periodic responses of a forced two degrees-of-freedom oscillatory system using nonlinear energy sink. Journal of Vibration and Control, 19(16):2401-2412, 2013. doi: 10.1177/ 1077546312456226. URL http://jvc.sagepub. com/content/19/16/2401. abstract.

S Bab, SE Khadem, MK Mahdiabadi, and
M Shahgholi. Vibration mitigation of a rotating beam under external periodic force using a nonlinear energy sink (nes). Journal of Vibration and Control, 2015. doi: 10.1177/1077546315587611. URL http://jvc.sagepub.com/content/early/ 2015/06/01/1077546315587611. abstract.

Saeed Bab, Siamak E. Khadem, and Majid Shahgholi. Lateral vibration attenuation of a rotor under mass eccentricity force using non-linear energy sink. International Journal of Non-Linear Mechanics, 67: 251-266, December 2014. ISSN 00207462. doi: 10.1016/j.ijnonlinmec.2014.08.016.

R. Bellet, B. Cochelin, P. Herzog, and P.-O. Mattei. Experimental study of targeted energy transfer from an acoustic system to a nonlinear membrane absorber. Journal of Sound and Vibration, 329:2768-2791, 2010.

B. Bergeot, S. Bellizzi, and B. Cochelin. Analysis of steady-state response regimes of a helicopter ground resonance model including a non-linear energy sink attachment. International Journal of Non-Linear Mechanics, 78: 72 - 89, 2016a. ISSN 0020-7462. doi: http: //dx.doi.org/10.1016/j.ijnonlinmec.2015.10.006. URL http://www.sciencedirect.com/science/ article/pii/S0020746215002000.

B. Bergeot, S. Bellizzi, and B. Cochelin. Passive suppression of helicopter ground resonance instability by means of a strongly nonlinear absorber. $A d$ vance in Aircraft and Spacecraft Science, 3(3):271298, 2016b.

C. Chevennement-Roux, T. Dreher, P. Alliot, E. Aubry, J.-P. Lainé, and L. Jézéquel. Flexible Wiper System Dynamic Instabilities: Modelling and Experimental Validation. Experimental Mechanics, 47(2):201-210, 2007. ISSN 0014-4851. doi: 10.1007/s11340-006-9027-3. URL http://link. springer. com/10.1007/s11340-006-9027-3.

M. Desroches, J. Guckenheimer, B. Krauskopf, C. Kuehn, H. Osinga, and M. Wechselberger. Mixed-mode oscillations with multiple time scales. SIAM Review, 52(2):211-288, 2012. doi: 10.1137/ 100791233.

E. Domany and O.V. Gendelman. Dynamic responses and mitigation of limit cycle oscillations in Van der Pol-Duffing oscillator with 
nonlinear energy sink. Journal of Sound and Vibration, 332(21):5489-5507, October 2013. ISSN 0022460X. doi: 10.1016/j.jsv.2013.05. 001. URL http://linkinghub.elsevier.com/ retrieve/pii/S0022460X13004069.

A.F. D'Souza and A.H. Dweib. Self-excited vibrations induced by dry friction, part 2: Stability and limit-cycle analysis. Journal of Sound and Vibration, 137(2):177-190, mar 1990. ISSN 0022460X. doi: 10.1016/0022-460X(90)90787-Z. URL http://www.sciencedirect.com/science/ article/pii/0022460X9090787Z.

M Eriksson and S Jacobson. Friction behaviour and squeal generation of disc brakes at low speeds. Proceedings of the Institution of Mechanical Engineers, Part D: Journal of Automobile Engineering, 215(12):1245-1256, 2001. ISSN 0954-4070. doi: 10.1243/0954407011528789. URL http://dx.doi. org/10.1243/0954407011528789.

Maor Farid and Oleg V Gendelman. Tuned pendulum as nonlinear energy sink for broad energy range. Journal of Vibration and Control, 2015. doi: 10.1177/1077546315578561. URL http://jvc.sagepub.com/content/early/ 2015/03/24/1077546315578561. abstract.

N Fenichel. Geometric singular perturbation theory for ordinary differential equations. Journal of Differential Equations, 98:53-98, 1979.

Guillaume Fritz, Jean-Jacques Sinou, Jean-Marc Duffal, and Louis Jézéquel. Investigation of the relationship between damping and mode-coupling patterns in case of brake squeal. Journal of Sound and Vibration, 307(3):591-609, 2007. ISSN 0022460X. doi: $10.1016 /$ j.jsv.2007.06.041.

O. V. Gendelman. Targeted energy transfer in systems with external and self-excitation. Proceedings of the Institution of Mechanical Engineers, Part C: Journal of Mechanical Engineering Science, 225(9): 2007-2043, 2011. doi: 10.1177/0954406211413976. URL http://pic.sagepub.com/content/225/9/ 2007 . abstract.

O. V. Gendelman and T. Bar. Bifurcations of selfexcitation regimes in a Van der Pol oscillator with a nonlinear energy sink. Physica D, 239(3-4):220229, February 2010. ISSN 01672789. doi: 10.1016/ j.physd.2009.10.020.
O.V. Gendelman, A.F. Vakakis, L.A. Bergman, and D.M. McFarland. Asymptotic analysis of passive nonlinear suppression of aeroelastic instabilities of a rigid wing in subsonic flow. SIAM Journal on Applied Mathematics, 70(5):1655-1677, 2010. doi: $10.1137 / 090754819$.

E. Gourc, S. Seguy, G. Michon, and A. Berlioz. Chatter Control in Turning Process with a Nonlinear Energy Sink. Advanced Materials Research, 698: 89-98, May 2013. ISSN 1662-8985. doi: 10.4028/ www.scientific.net/AMR.698.89.

E. Gourdon, N. A. Alexander, C. A. Taylor, C. H. Lamarque, and S. Pernot. Nonlinear energy pumping under transient forcing with strongly nonlinear coupling: Theoretical and experimental results. Journal of Sound and Vibration, 300(3-5):522-551, 2007. ISSN 10958568. doi: 10.1016/j.jsv.2006.06. 074 .

J. Grasman. Asymptotic Methods for Relaxation Oscillations and Applications, volume 63 of Applied Mathematical Sciences. Springer-Verlag, 1987.

B. Hervé, J.-J. Sinou, H. Mahé, and L. Jézéquel. Analysis of squeal noise and mode coupling instabilities including damping and gyroscopic effects. European Journal of Mechanics A/Solids, 27(2):141-160, mar 2008. ISSN 09977538. doi: 10.1016/j.euromechsol.2007.05.004. URL http://www.sciencedirect.com/science/ article/pii/S0997753807000447.

$\mathrm{N}$ Hoffmann and L Gaul. Effects of damping on mode-coupling instability in friction induced oscillations. $\quad\{Z A M M\}$ - Journal of Applied Mathematics and Mechanics / Zeitschrift für Angewandte Mathematik und Mechanik, 83(8):524-534, 2003. ISSN 1521-4001. doi: 10.1002/zamm. 200310022. URL http://onlinelibrary.wiley. com/doi/10.1002/zamm. 200310022/abstract.

J. Hultén. Brake squeal - a self-exciting mechanism with constant friction. In SAE Truck and Bus Meeting, Detroit, Mi, USA, 1993.

J. Hultén. Friction phenomena related to drum brake squeal instabilities. In ASME Design Engineering Technical Conferences, Sacramento, CA, 1997.

ChristopherK.R.T. Jones. Geometric singular perturbation theory. In Russell Johnson, editor, Dynamical Systems, volume 1609 of Lecture Notes in 
Mathematics, pages 44-118. Springer Berlin Heidelberg, 1995. ISBN 978-3-540-60047-3. doi: 10.1007/ BFb0095239.

Gaëtan Kerschen, D. Michael McFarland, Jeffrey J. Kowtko, Young S. Lee, Lawrence A. Bergman, and Alexander F. Vakakis. Experimental demonstration of transient resonance capture in a system of two coupled oscillators with essential stiffness nonlinearity. Journal of Sound and Vibration, 299(4-5):822-838, 2007. ISSN 10958568. doi: 10.1016/j.jsv.2006.07.029.

Y. S. Lee, A. F. Vakakis, L. A. Bergman, and D. M. McFarland. Suppression of limit cycle oscillations in the van der Pol oscillator by means of passive nonlinear energy sinks. Structural Control and Health Monitoring, 13(1):41-75, January 2006. ISSN 15452255. doi: $10.1002 /$ stc. 143 .

Y. S. Lee, A. F. Vakakis, L. A. Bergman, D. M. McFarland, and G. Kerschen. Suppression aeroelastic instability using broadband passive targeted energy transfers, part 1: Theory. AIAA Journal, 45 (3):693-711, March 2007a. ISSN 0001-1452. doi: $10.2514 / 1.24062$.

Y. S. Lee, A. F. Vakakis, L. A. Bergman, D. M. McFarland, and G. Kerschen. Suppression aeroelastic instability using broadband passive targeted energy transfers, part 2: Experiments. AIAA Journal, 45 (3):2391-2400, March 2007b. ISSN 0001-1452. doi: $10.2514 / 1.24062$.

Angelo Luongo and Daniele Zulli. Aeroelastic instability analysis of nes-controlled systems via a mixed multiple scale/harmonic balance method. Journal of Vibration and Control, 2013. doi: 10.1177/1077546313480542. URL http://jvc.sagepub.com/content/early/ 2013/06/17/1077546313480542.abstract.

L.I. Manevitch. Complex representation of dynamics of coupled nonlinear oscillators. In LudmilaA. Uvarova, ArkadiiE. Arinstein, and AnatoliiV. Latyshev, editors, Mathematical Models of Non-Linear Excitations, Transfer, Dynamics, and Control in Condensed Systems and Other Media, pages 269-300. Springer US, 1999. doi: 10.1007/ 978-1-4615-4799-0 $\_24$.

L. Nechak, S. Berger, and E. Aubry. Non-intrusive generalized polynomial chaos for the robust stabil- ity analysis of uncertain nonlinear dynamic friction systems. Journal of Sound and Vibration, 332(5):1204-1215, 2013. ISSN 0022460X. doi: 10.1016/j.jsv.2012.09.046.

J.T. Oden and J.A.C. Martins. Models and computational methods for dynamic friction phenomena. Computer Methods in Applied Mechanics and Engineering, 52(1):527-634, 1985. ISSN 00457825. doi: 10.1016/0045-7825(85)90009-X.

J.-J. Sinou, O. Dereure, G.-B. Mazet, F. Thouverez, and L. Jezequel. Friction-induced vibration for an aircraft brake system-Part 1: Experimental approach and stability analysis. International Journal of Mechanical Sciences, 48(5):536-554, may 2006a. ISSN 00207403. doi: 10.1016/j.ijmecsci. 2005.12.002. URL http://www.sciencedirect. com/science/article/pii/S0020740305002936.

J.-J. Sinou, F. Thouverez, L. Jezequel, O. Dereure, and G.-B. Mazet. Friction induced vibration for an aircraft brake system-Part 2: Non-linear dynamics. International Journal of Mechanical Sciences, 48(5):555-567, may 2006b. ISSN 00207403. doi: 10.1016/j.ijmecsci.2005.12.003. URL http://www.sciencedirect.com/science/ article/pii/S0020740305002948.

Jean Jacques Sinou and Louis Jézéquel. Mode coupling instability in friction-induced vibrations and its dependency on system parameters including damping. European Journal of Mechanics, A/Solids, 26(1):106-122, 2007. ISSN 09977538. doi: 10.1016/j.euromechsol.2006.03.002.

Y. Starosvetsky and O. V. Gendelman. Strongly modulated response in forced 2dof oscillatory system with essential mass and potential asymmetry. Physica D, 237(13):1719-1733, August 2008. ISSN 01672789. doi: 10.1016/j.physd.2008.01.019.

A.F. Vakakis and O.V. Gendelman. Energy pumping in nonlinear mechanical oscillators: Part II - Resonance capture. Journal of Applied Mechanics, 68: 42-48, 2001.

A. F. Vakatis, O. V. Gendelman, L. A. Bergman, D. M. McFarland, G. Kerschen, and Y. S. Lee. Nonlinear Targeted Energy Transfer in Mechanical and Structural Systems. Springer-Verlag, Berlin, New York, 2008. ISBN 9781402091254. 
F. Van De Velde and P. De Baets. The relation between friction force and relative speed during the slip-phase of a stick-slip cycle. Wear, 219(2):220-226, sep 1998a. ISSN 00431648. doi: 10.1016/S0043-1648(98)00213-0. URL http://www.sciencedirect.com/science/ article/pii/S0043164898002130.

F. Van De Velde and P. De Baets. A new approach of stick-slip based on quasi-harmonic tangential oscillations. Wear, 216(1):15-26, mar 1998b. ISSN 00431648. doi: 10.1016/S0043-1648(97)00296-2. URL http://www.sciencedirect.com/science/ article/pii/S0043164897002962.

Stephen Wiggins. Introduction to Applied Nonlinear Dynamical Systems and Chaos. Texts in Applied Mathematics 2. Springer New York, 2nd ed edition, 1990. ISBN 9780387001777,0387001778. URL http://gen.lib.rus.ec/book/index.php? md5=FC5B75B65E2F01A76E888A8082C8D63C. 


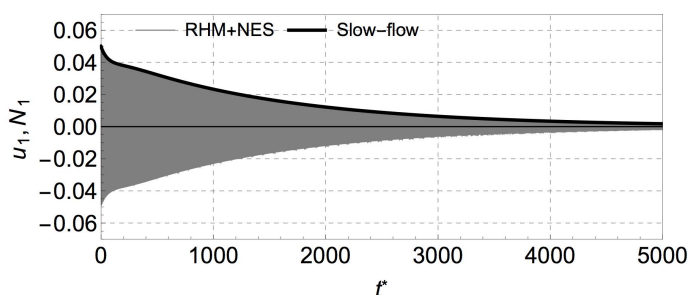

(a)

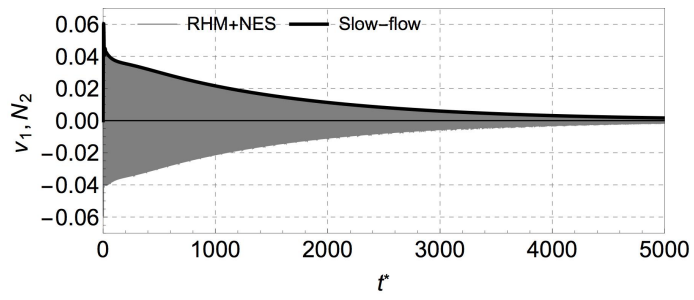

(c)

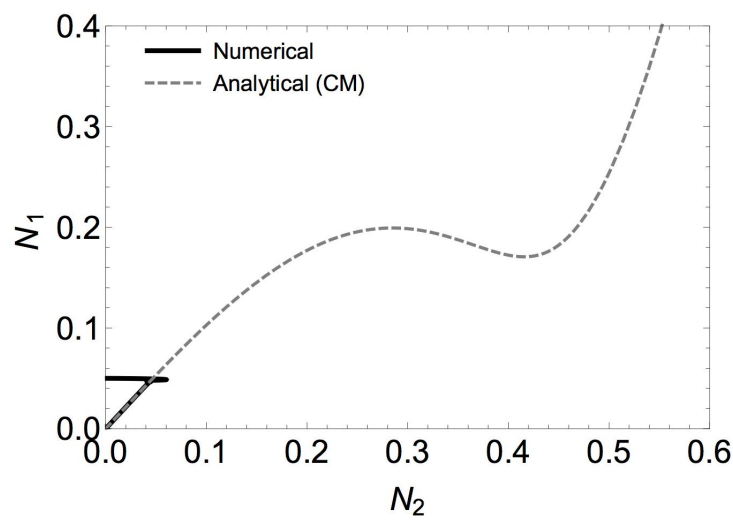

(e)

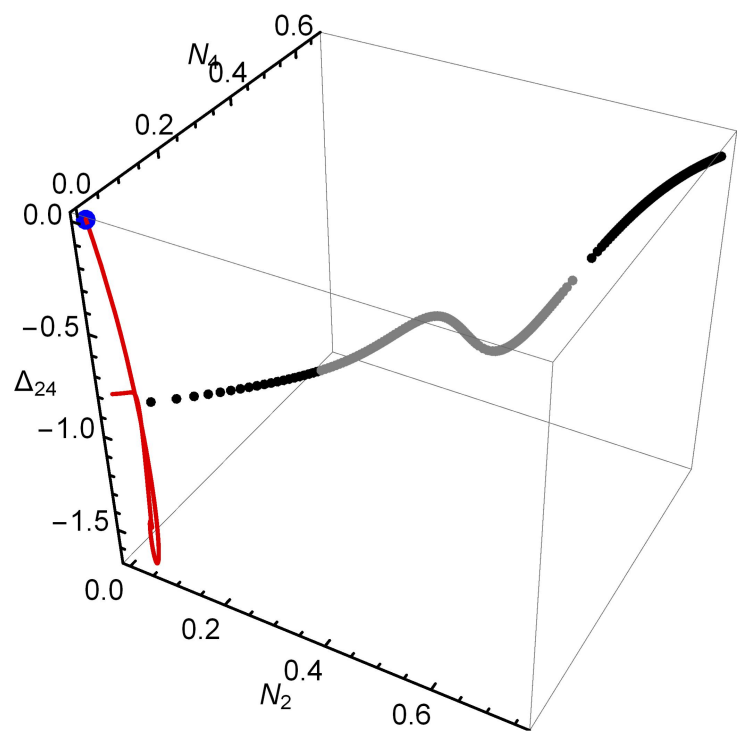

(g)

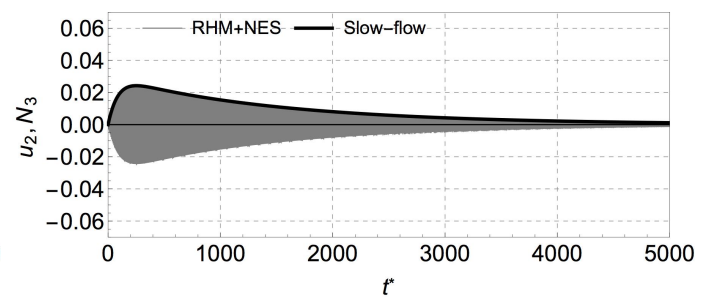

(b)

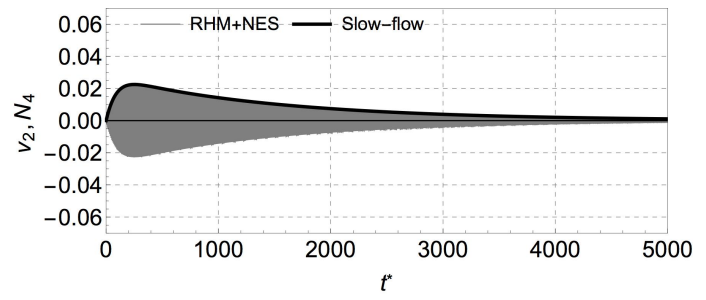

(d)

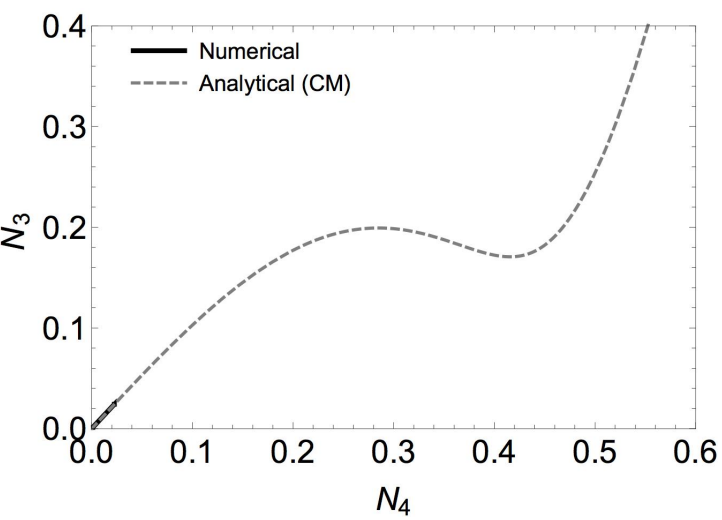

(f)

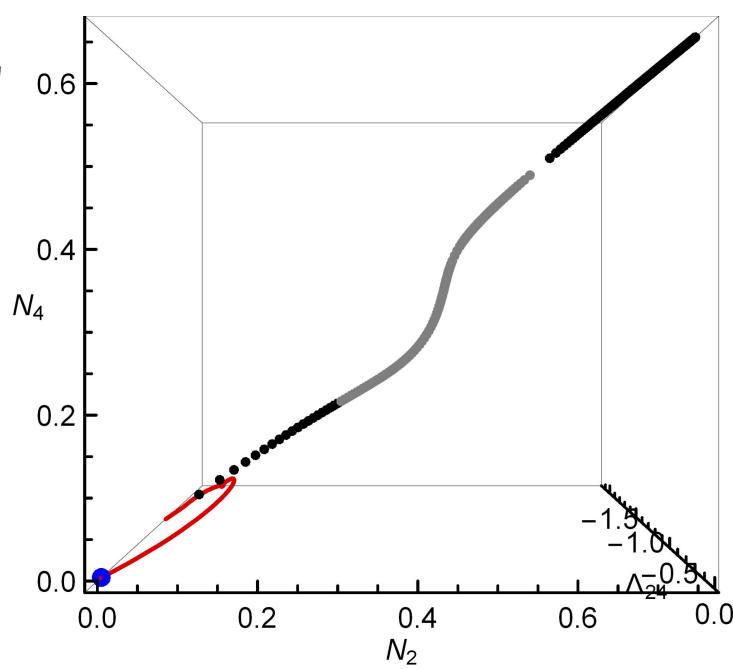

(h)

Figure 10: (a), (b), (c) and (d) compare numerical simulations of the RHM+NES written using barycentric coordinates (see Eq. (17)) (solid grey line) and numerical simulations of the slow-flow (21) (solid black line). (e) and (f) compare the Critical Manifold $N_{1}=\sqrt{H\left(N_{2}\right)}$ and $N_{3}=\sqrt{H\left(N_{4}\right)}$ (dashed grey line) (see Eq. (32)) with numerical simulations of the slow-flow (solid black line). (g) and (h) compare the bifurcation diagram obtained from Eq. (53) (black and grey points for stable and unstable branches respectively) with the trajectory of the slow-flow (solid red line) in the 3D $N_{2}, N_{4}, \Delta_{25}$-space, The blue point shows the initial condition and two views are used to make the reading of the $3 \mathrm{D}$ graph easier. Set of parameters (10) is used and $\sigma=0.2$. 


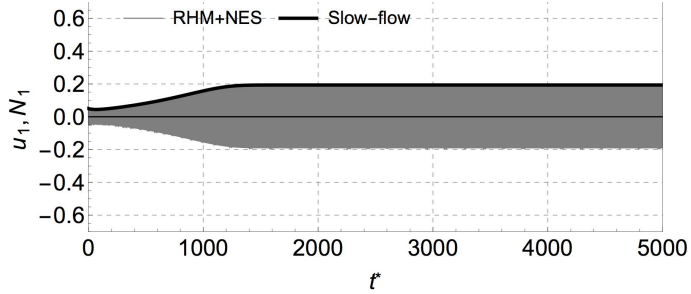

(a)

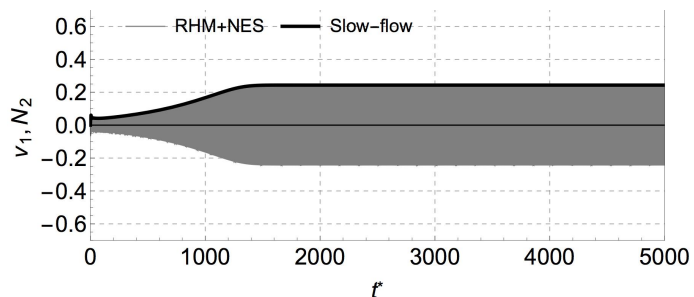

(c)

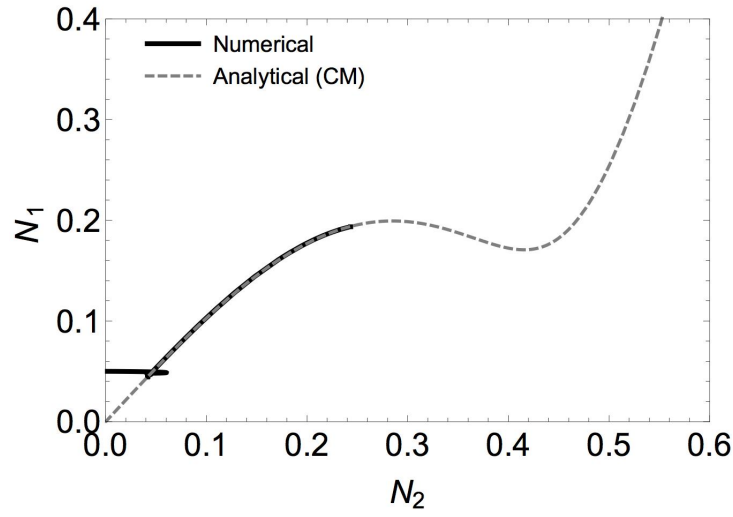

(e)

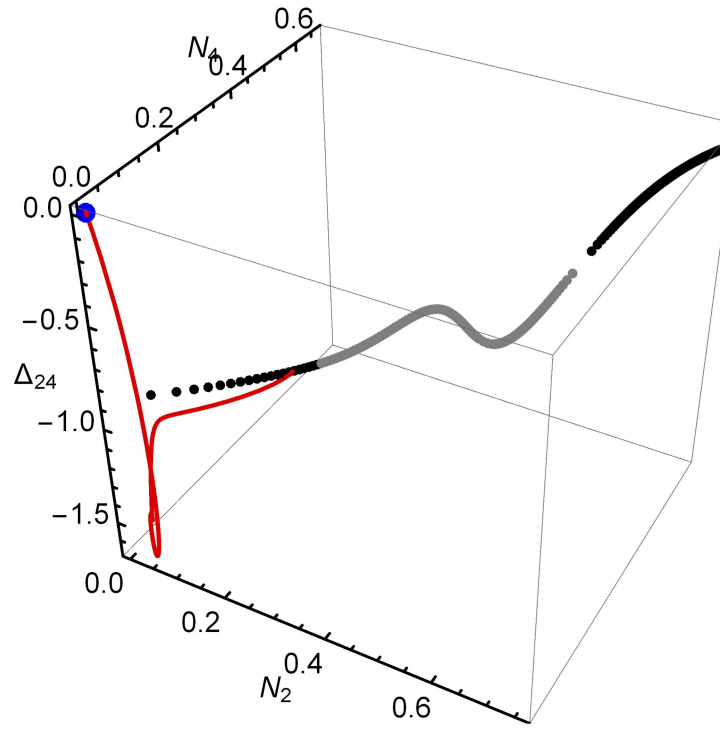

(g)

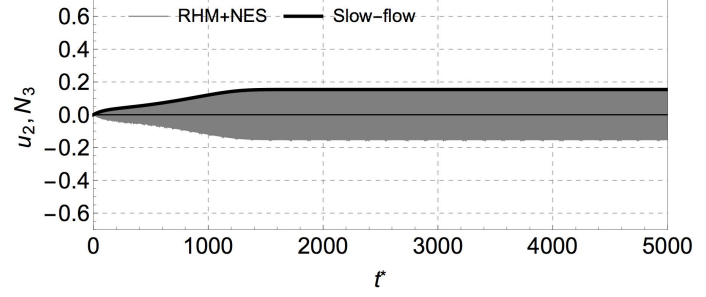

(b)

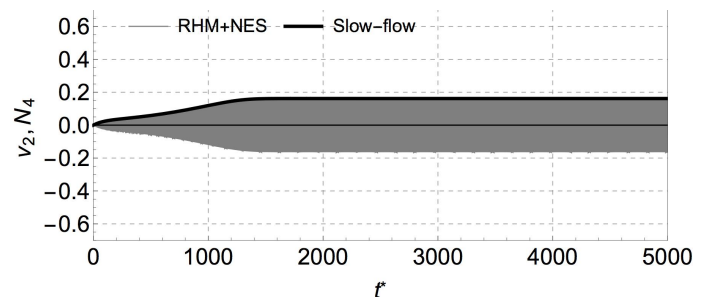

(d)

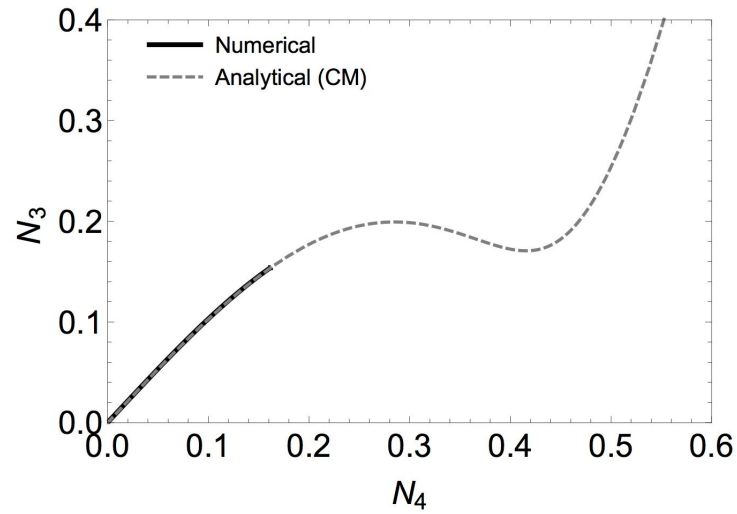

(f)

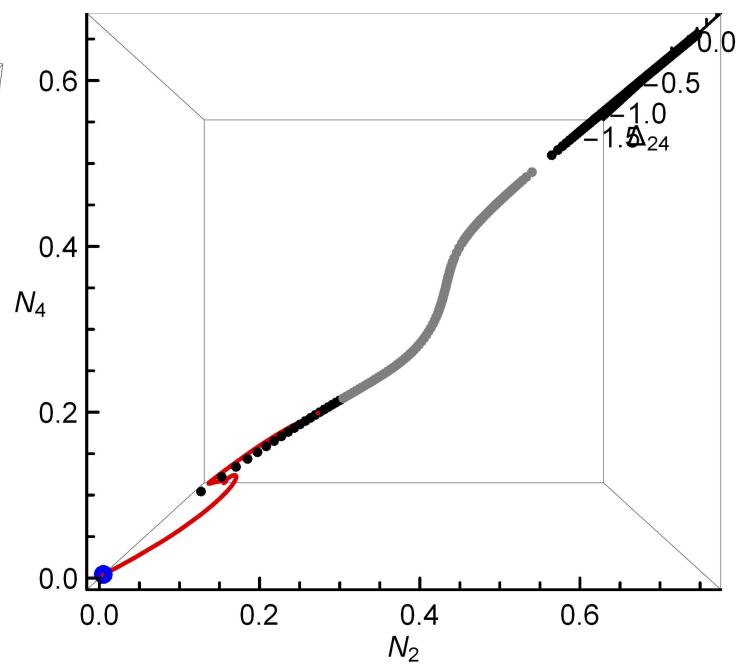

(h)

Figure 11: Same caption as for Fig. 10. Set of parameters (10) is used and $\sigma=0.6$. 


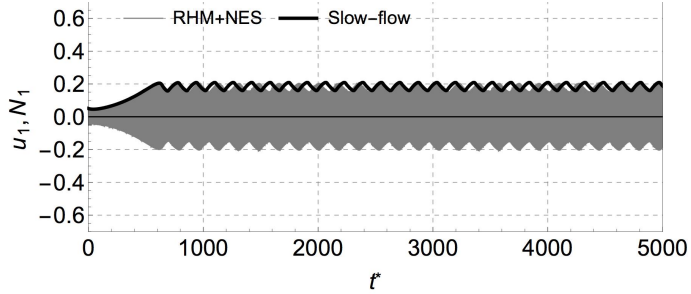

(a)

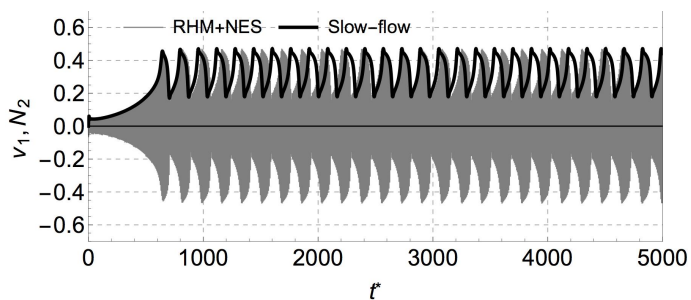

(c)

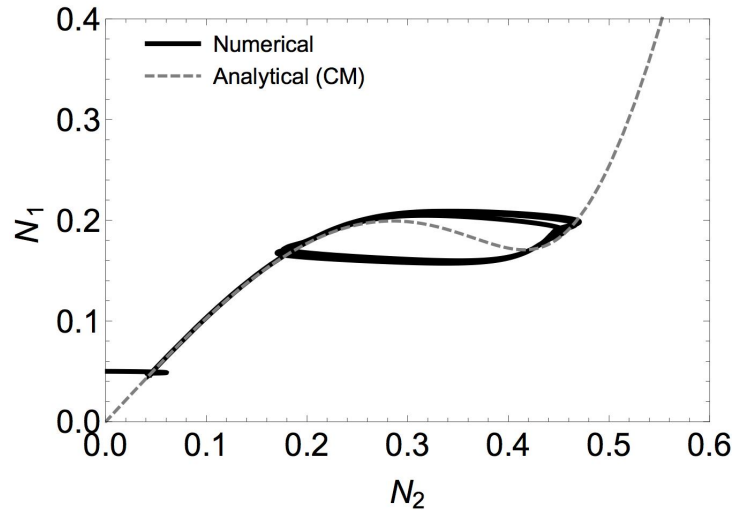

(e)

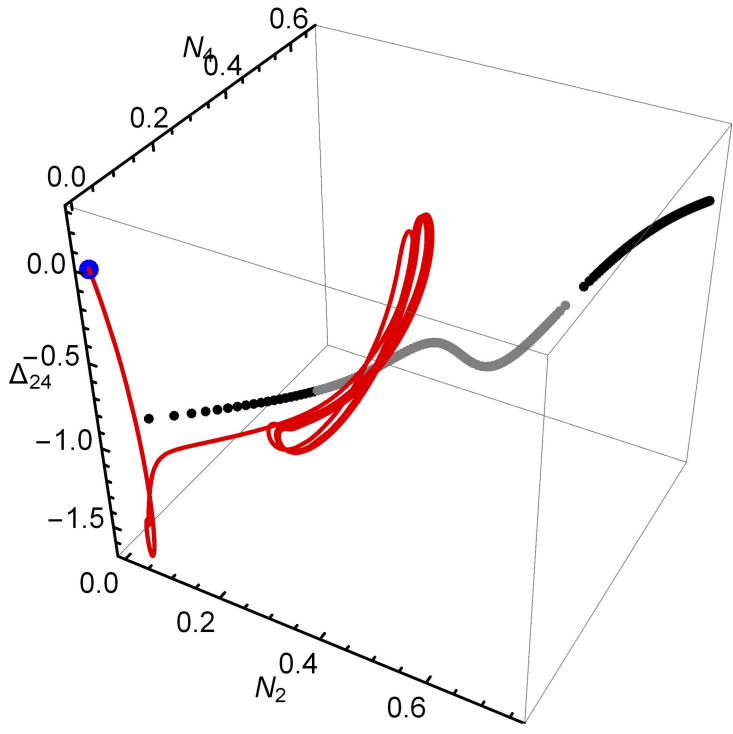

(g)

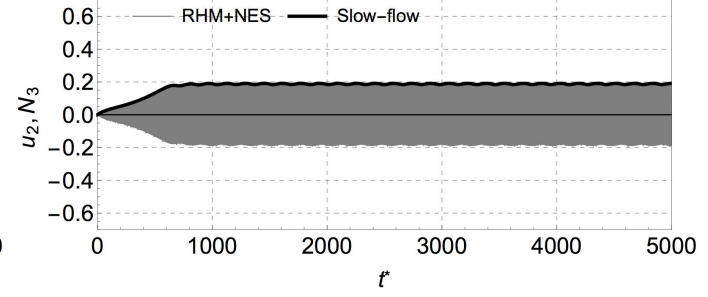

(b)

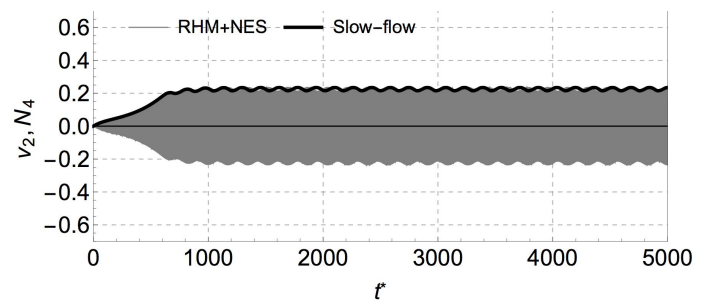

(d)

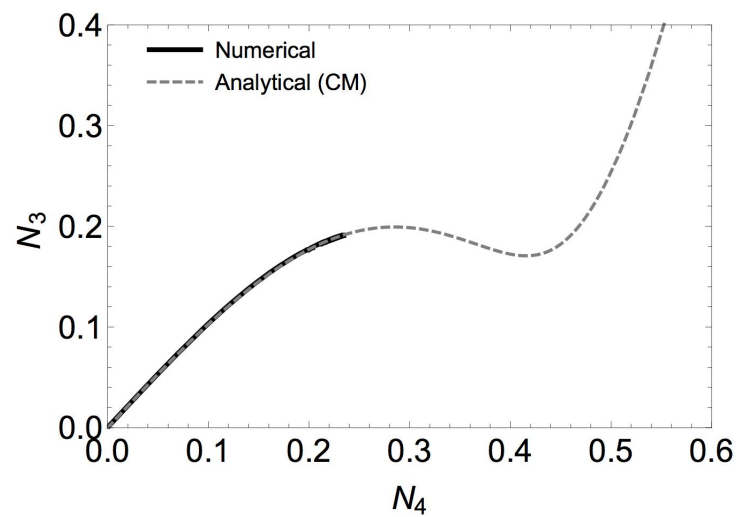

(f)

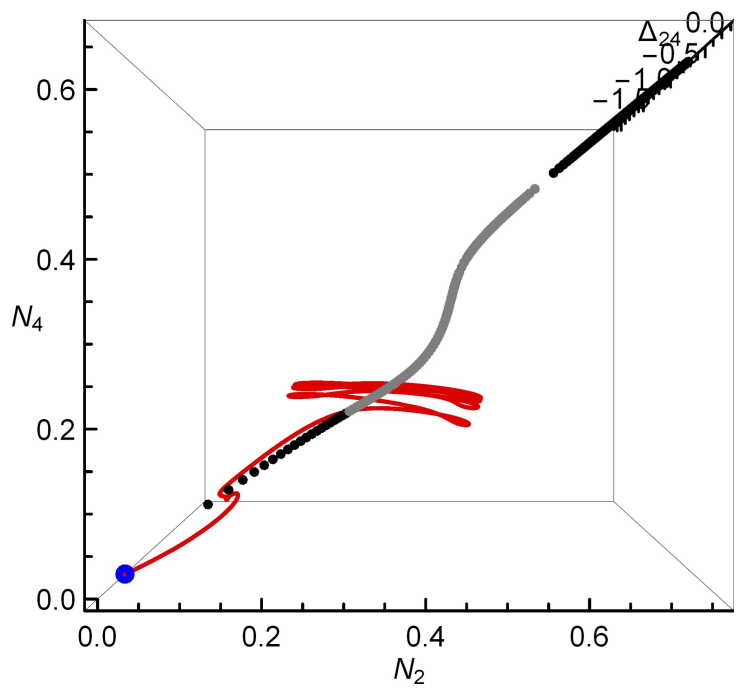

(h)

Figure 12: Same caption as for Fig. 10. Set of parameters (10) is used and $\sigma=0.9$. 


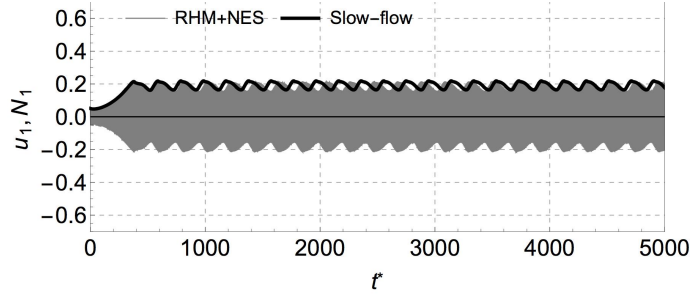

(a)

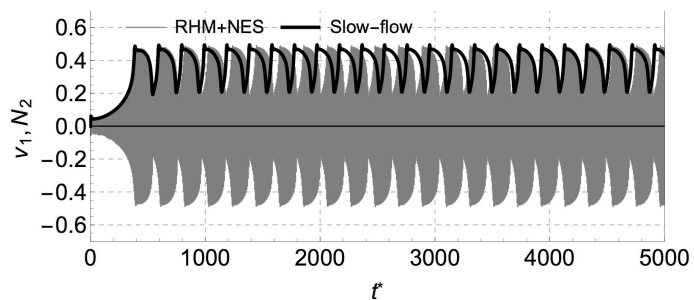

(c)

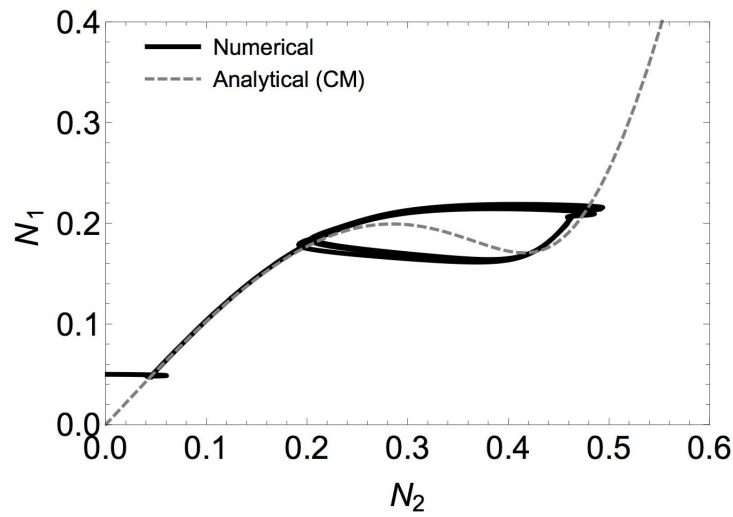

(e)

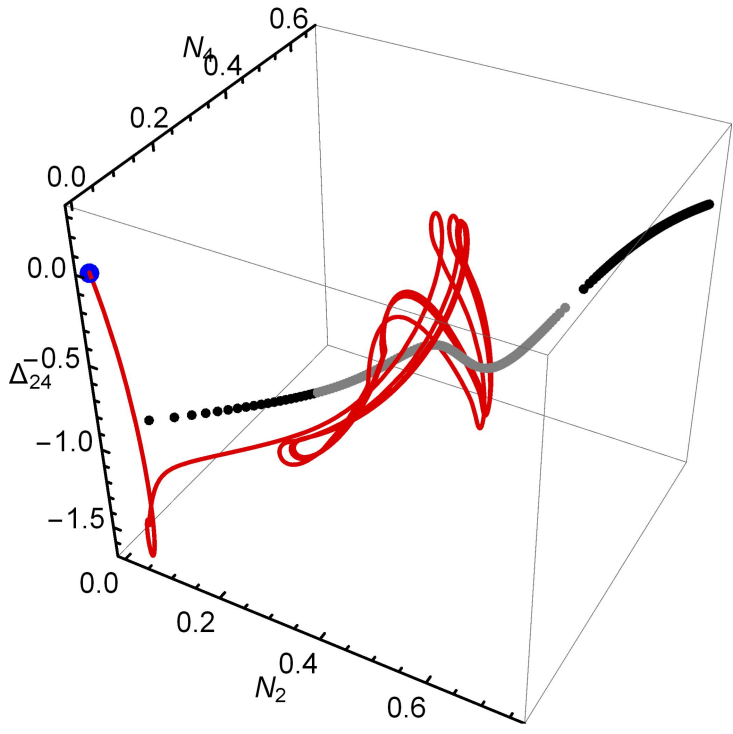

(g)

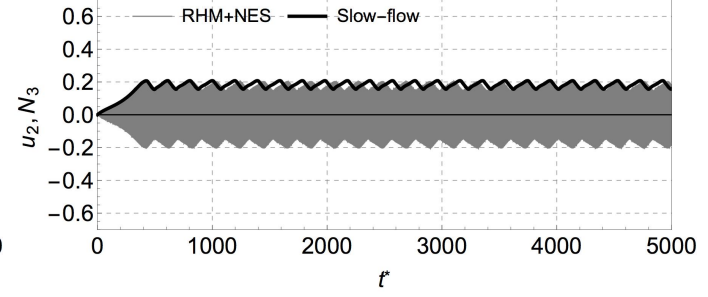

(b)

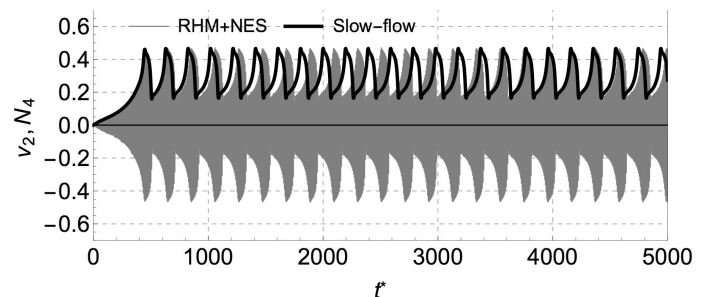

(d)

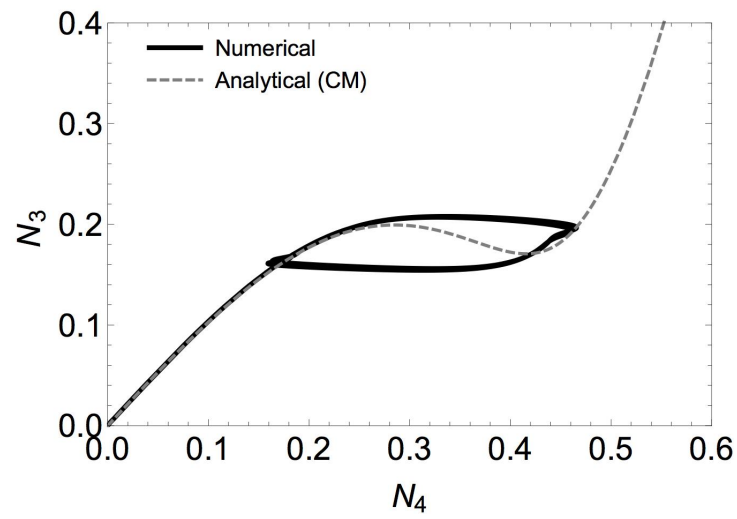

(f)

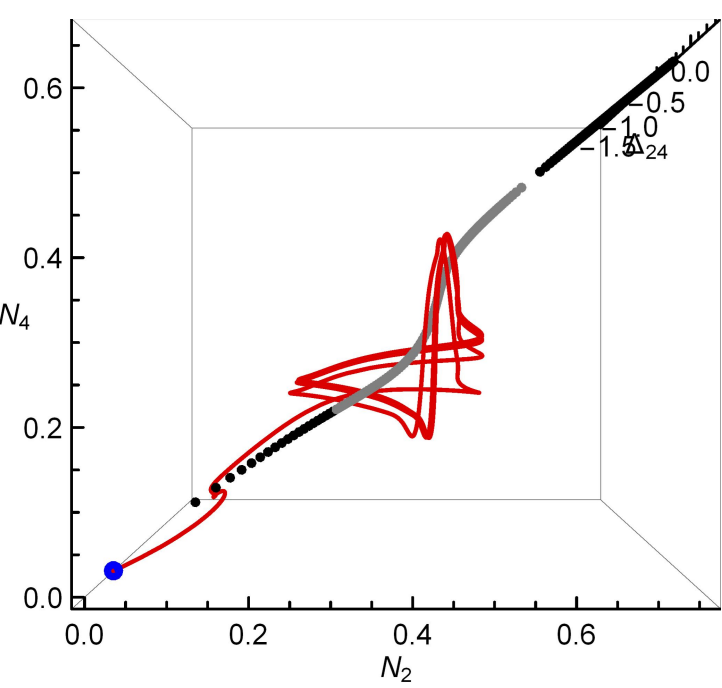

(h)

Figure 13: Same caption as for Fig. 10. Set of parameters (10) is used and $\sigma=1.3$. 


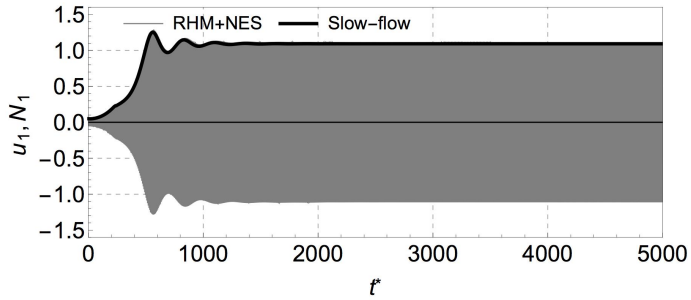

(a)

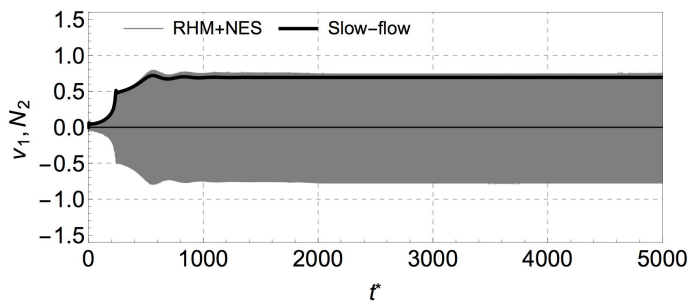

(c)

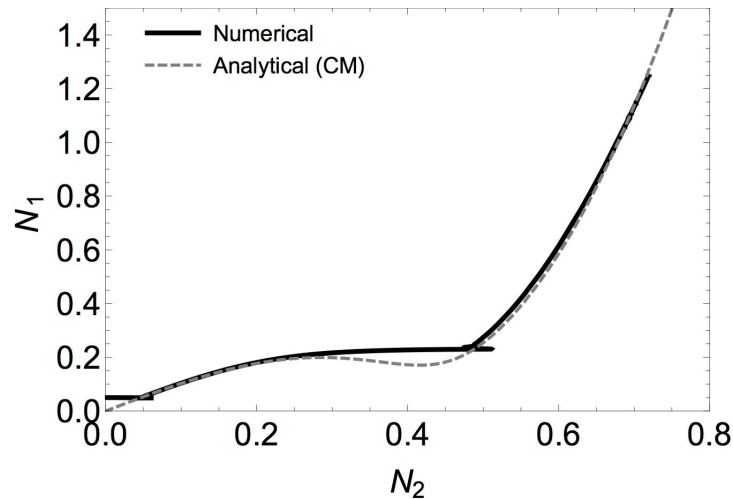

(e)

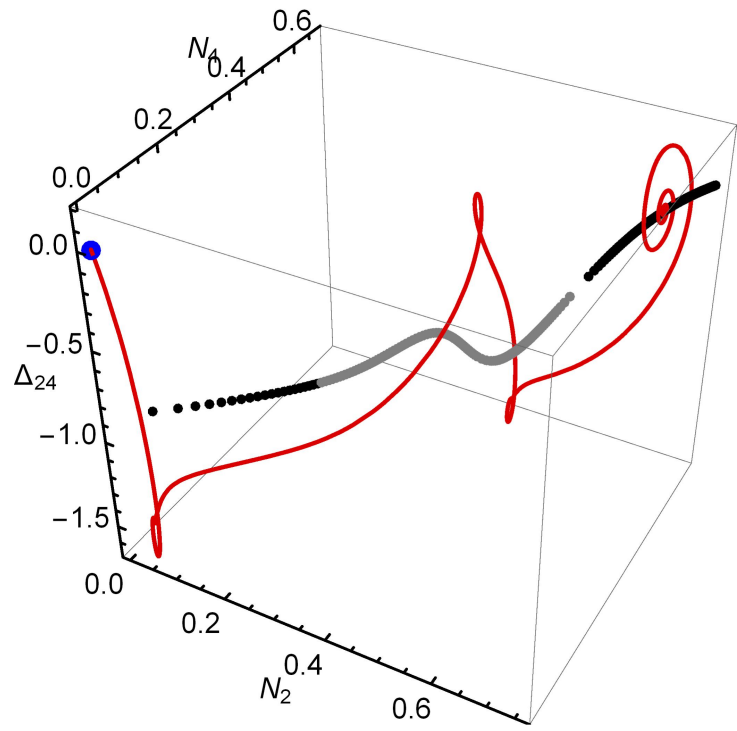

(g)

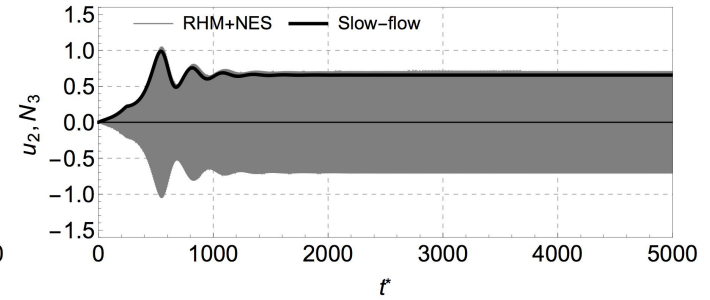

(b)

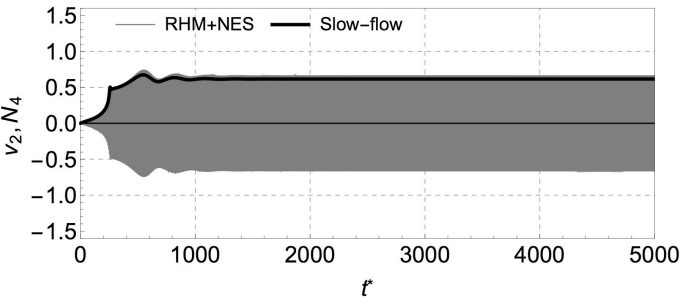

(d)

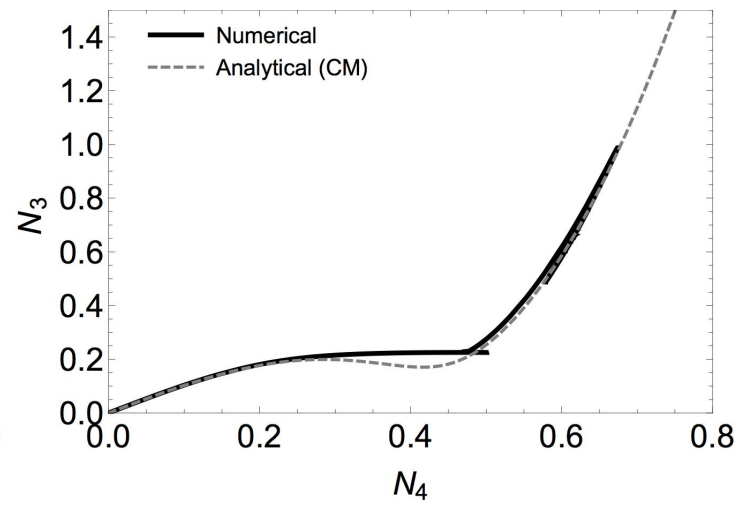

(f)

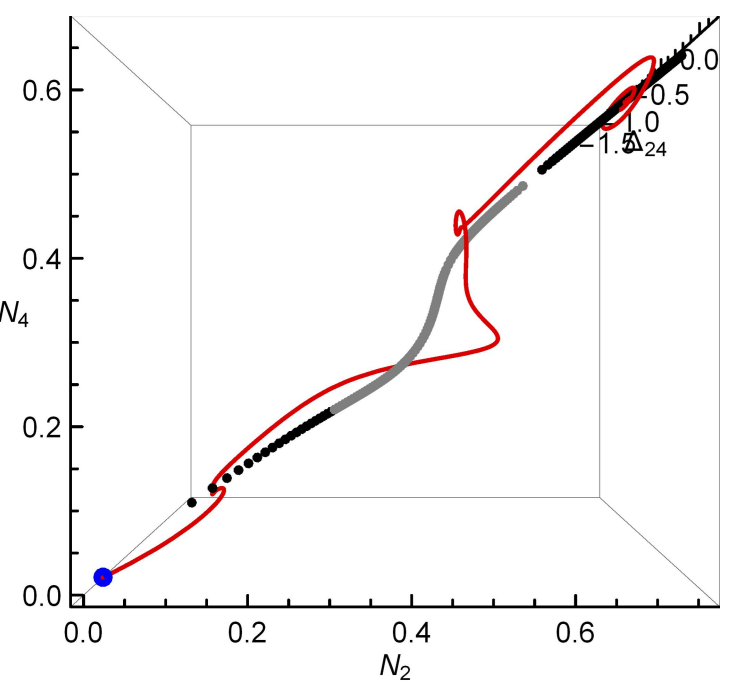

(h)

Figure 14: Same caption as for Fig. 10. Set of parameters (10) is used and $\sigma=2$. 


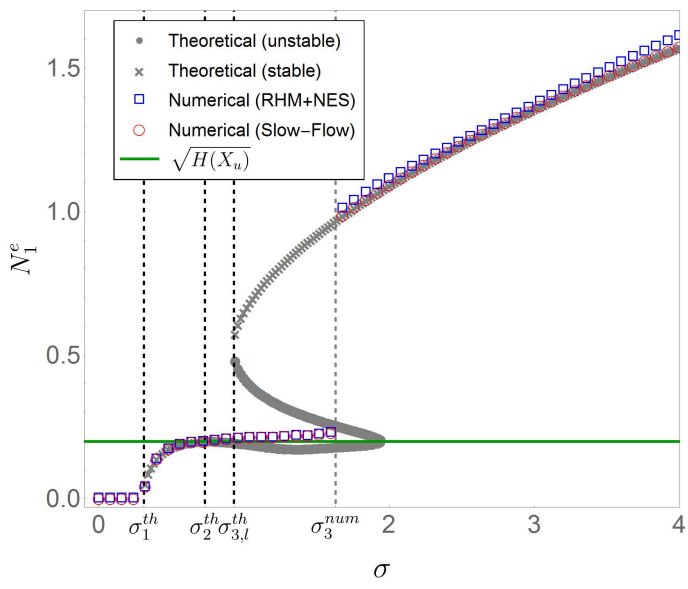

(a)

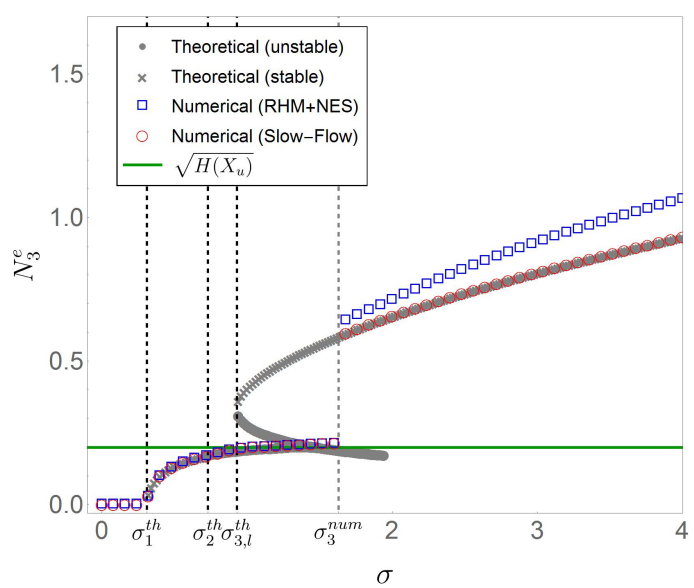

(c)

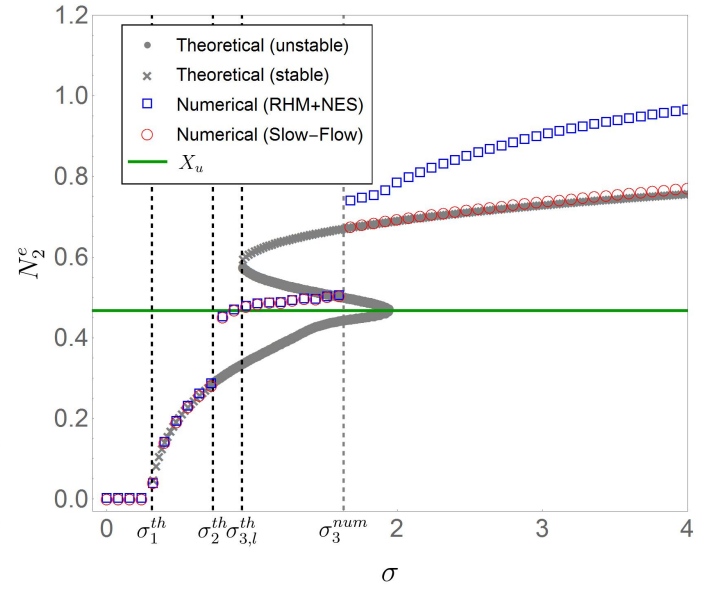

(b)

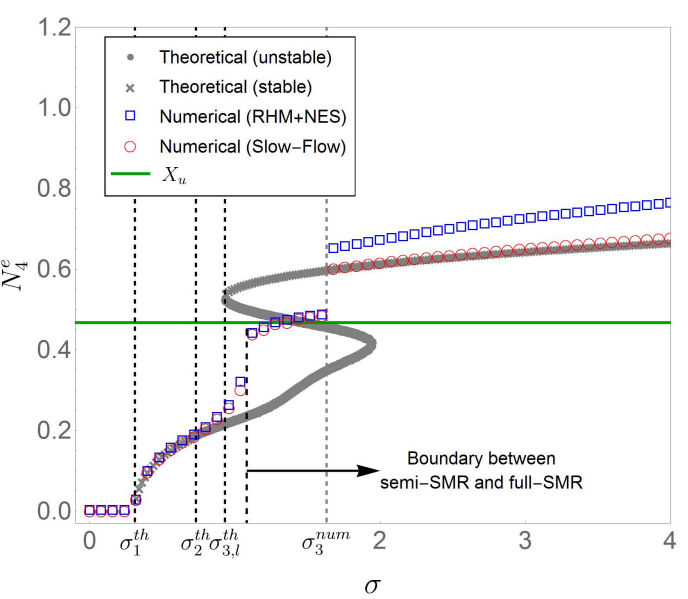

(d)

Figure 15: Comparison between steady-state amplitudes obtained from numerical simulations of the RHM+NES written using barycentric coordinates (see Eq. (17)) (blue squares) and from numerical simulations of the slow-flow (21) (red circles). Set of parameters (10) is used. 